\title{
Plünnecke and Kneser type theorems for dimension estimates
}

\author{
Cédric Lecouvey
}

\begin{abstract}
Given a division ring $K$ containing the field $\mathrm{k}$ in its center and two finite subsets $A$ and $B$ of $K^{*}$, we give some analogues of Plünnecke and Kneser Theorems for the dimension of the k-linear span of the Minkowski product $A B$ in terms of the dimensions of the k-linear spans of $A$ and $B$. We also explain how they imply the corresponding more classical theorems for abelian groups. These Plünnecke type estimates are then generalized to the case of associative algebras. We also obtain an analogue in the context of division rings of a theorem by Tao describing the sets of small doubling in a group. AMS classification: 05E15, 12E15, 11P70.

Keywords: division ring, Kneser's theorem, Plünnecke-Ruzsa's inequalities.
\end{abstract}

\section{Introduction}

A classical problem in additive number theory is to evaluate the cardinality of sumsets in $\mathbb{Z}$ in terms of the cardinality of their summands. Many results and methods used to obtain such evaluations are in fact also suited for studying the cardinality of any sumset in abelian groups. In this paper, we will write group operations multiplicatively. Among numerous interesting results in this area are the Plünnecke-Ruzsa and Kneser theorems.

Plünnecke-Ruzsa's theorem gives an upper bound for the cardinality of $A^{n}$ knowing such a bound for $\left|A^{2}\right|$.

Theorem 1.1 Let $A$ and $B$ be two finite subsets in an abelian group $G$. Assume $\alpha$ is a positive real such that $|A B| \leq \alpha|A|$. Then there exists a subset $X$ of $A$ such that, for any integer $n$, $\left|X B^{n}\right| \leq \alpha^{n}|X|$. In particular $\left|A^{2}\right| \leq \alpha|A|$ implies that $\left|A^{n}\right| \leq \alpha^{n}|A|$.

Kneser's theorem gives a lower bound for the cardinality of the product set $A B$ where $A$ and $B$ are finite nonempty subsets in an abelian group $G$.

Theorem 1.2 Let $A$ and $B$ be finite subsets of the abelian group $G$. Write $H$ for the stabilizer of $A B$ in $G$. Then

$$
|A B| \geq|A|+|B|-|H|
$$

It is then natural to ask for analogous results in the general case of possibly non abelian groups. The question of finding lower and upper bounds for product sets in non abelian groups is considerably more difficult than in the abelian case. Nevertheless, there is a growing literature on this subject due to Diderrich [2, Hamidoune [6], Kemperman [11, Olson [15], Ruzsa [17], Tao [20] and many others. Let us mention that Kneser's theorem does not hold for non abelian groups as noticed in [11. Nevertheless, there exist in this case weaker versions due to Diderrich [2] and Olson [15. 
The Plünnecke-Ruzsa and Kneser theorems give informations on the structure of groups. In this paper, we establish analogous results in the context of division rings. According to the usual terminology, a field is a commutative division ring. It is very easy to produce fields as extensions of simpler ones. Recall that Representation Theory of groups gives also a general procedure yielding division rings which are not necessarily fields. Indeed, given a field $\mathrm{k}$ and a group $G$, the Schur lemma implies that the endomorphism algebra $K$ of any irreducible finite-dimensional $\mathrm{k}[G]$-module ( $\mathrm{k}[G]$ is the ring algebra of $G$ over $\mathrm{k}$ ) is a division ring. When $\mathrm{k}$ is not algebraically closed, $K \supsetneqq \mathrm{k}$ and is not commutative in general. So incidentally, we will obtain structural information on the division ring of automorphisms of any finite-dimensional $\mathrm{k}[G]$-module.

Let us make our notation precise. In what follows, $K$ is a division ring containing the field $\mathrm{k}$ in its center. We address the question of finding upper and lower bounds for the dimension of the k-span $\mathrm{k}\left\langle A_{1} \cdots A_{n}\right\rangle$ of product sets $A_{1} \cdots A_{n}$, where $A_{1}, \ldots, A_{n}$ are nonempty subsets of $K^{*}=K \backslash\{0\}$. Note that this problem also makes sense for any algebra $\mathcal{A}$ defined over k. The estimates we obtain can thus also be applied when $\mathcal{A}$ is contained in a division ring. In the commutative case, this happens in particular for any algebra $\mathcal{A}:=\mathrm{k}\left[\alpha_{1}, \ldots, \alpha_{n}\right]$, where $\alpha_{1}, \ldots, \alpha_{n}$ are elements of the field $K$, or for any sub-algebra of a field of rational functions in several variables.

As far as we are aware, this kind of problems was considered for the first time by Hou, Leung and Xiang [4], and Kainrath [10]. Let $X$ be a finite subset in $K$. We denote by $\mathrm{k}\langle X\rangle$ the ksubspace of $K$ generated by $X$ and write $\operatorname{dim}_{\mathrm{k}}(X)$ its dimension. For $X, Y$ two subsets of $K$, we set $X Y=\{x y \mid x \in X, y \in Y\}$. Consider $A, B$ finite subsets of $K$. Then $\mathrm{k}\langle A B\rangle=\mathrm{k}\langle\mathrm{k}\langle A\rangle \mathrm{k}\langle B\rangle\rangle$ and $\operatorname{dim}_{\mathrm{k}}(A B)$ is finite. The following analogue of Kneser's theorem for fields is proved in [4].

Theorem 1.3 Let $K$ be a commutative extension of $\mathrm{k}$. Assume every algebraic element in $K$ is separable over k. Let $A$ and $B$ be two nonempty finite subsets of $K^{*}$. Then

$$
\operatorname{dim}_{\mathrm{k}}(A B) \geq \operatorname{dim}_{\mathrm{k}}(A)+\operatorname{dim}_{\mathrm{k}}(B)-\operatorname{dim}_{\mathrm{k}}(H)
$$

where $H:=\{x \in K \mid x \mathrm{k}\langle A B\rangle \subseteq \mathrm{k}\langle A B\rangle\}$.

Here $H$ is an intermediate field containing k. Remarkably, the authors showed that their theorem implies Kneser's theorem for abelian groups. It essentially suffices to use the characterization of all finitely generated abelian groups and the Galois correspondence. In [3], we obtain an analogue of a theorem by Olson for division rings without any separability hypothesis. In what follows, we shall refer to these analogues as linear Kneser and linear Olson theorems. The combinatorial methods used in the linear setting (that is, for fields or division rings) are often very similar to their counterparts in groups. Nevertheless, there are some restrictions and complications mostly due to the fact that

- $\mathrm{k}\langle A\rangle$ and $\mathrm{k}\left\langle A^{-1}\right\rangle$ do not have the same dimension in general, whereas $A$ and $A^{-1}$ have the same cardinality,

- a k-subspace $V$ in $K$ may admit infinitely many k-subspaces $W$ such that $V \oplus W=K$, whereas a subset $A$ in a group $G$ has a unique complement,

- when $K$ is finite-dimensional over k, there may exist infinitely many intermediate division rings $H$ such that $\mathrm{k} \subseteq H \subseteq K$, whereas a finite group $G$ has only a finite number of subgroups,

- given $H_{1}$ and $H_{2}$ subfields of the (commutative field) $K, H_{1} H_{2}$ is not a field in general, whereas the product set of two subgroups of an abelian group is always a group. 
So, to avoid gaps or ambiguities, we have completely written down the proofs of our linear statements. These proofs sometimes differ from their analogues in groups. For example, the possible existence of an infinite number of intermediate fields seems to impose a separability hypothesis in the previous linear Kneser theorem. It is nevertheless conjectured in [9] that this hypothesis can be relaxed as in the linear Olson theorem. Also, in order to adapt the arguments used to establish the estimates in groups, we often need, in our division ring context, to carefully chose the decomposition in direct summands of the spaces we consider in our proofs.

The paper is organized as follows. In Section 2, we make our notation precise and give equivalent forms of the linear Kneser theorem. Section 3 is devoted to some linear analogues of results by Ruzsa. In particular, we derive a Plünnecke-Ruzsa type theorem for fields. The arguments we use here are adaptations to the context of division rings of some very elegant and elementary proofs recently obtained by Petridis in [16]. We also establish that our Plünnecke-Ruzsa theorem for fields implies the corresponding theorem for abelian groups. In Section 4, we generalize the Plünnecke-Ruzsa type theorems of Section 2 in the context of associative unital algebras. In Section 5, we establish different Kneser type estimates for division rings. More precisely, we first study the case where $A$ is assumed commutative (that is, the elements of $A$ are pairwise commutative) and obtain a linear analogue of a theorem by Diderrich [2]. Finally, we adapt Hamidoune connectivity to the context of division rings and obtain a linear version of a theorem by Tao describing the sets of small doubling in a group.

\section{The division ring setting}

\subsection{Vector span in a division ring}

Let $K$ be a division ring and $\mathrm{k}$ a subfield (thus commutative) of $K$ contained in its center. We denote by $K^{*}=K \backslash\{0\}$ the group of invertible elements in $K$.

For any subset $A$ of $K^{*}$, let $\mathrm{k}\langle A\rangle$ be the k-subspace of $K$ generated by $A$. We write $|A|$ for the cardinality of $A$, and $\operatorname{dim}_{\mathrm{k}}(A)$ for the dimension of $\mathrm{k}\langle A\rangle$ over $\mathrm{k}$. When $|A|$ is finite, $\operatorname{dim}_{\mathrm{k}}(A)$ is also finite and we have $\operatorname{dim}_{\mathrm{k}}(A) \leq|A|$. We denote by $\mathbb{D}(A) \subseteq K$ the sub division ring generated by $A$ in $K$.

Given subsets $A$ and $B$ of $K$, we thus have $\mathrm{k}\langle A \cup B\rangle=\mathrm{k}\langle A\rangle+\mathrm{k}\langle B\rangle$, the sum of the two spaces $\mathrm{k}\langle A\rangle$ and $\mathrm{k}\langle B\rangle$. We have also $\mathrm{k}\langle A \cap B\rangle \subseteq \mathrm{k}\langle A\rangle \cap \mathrm{k}\langle B\rangle$ and $\mathrm{k}\langle A B\rangle=\mathrm{k}\langle\mathrm{k}\langle A\rangle \mathrm{k}\langle B\rangle\rangle$. We write as usual

$$
A B:=\{a b \mid a \in A, b \in B\}
$$

for the Minkowski product of the sets $A$ and $B$. Given a family of nonempty subsets $A_{1}, \ldots, A_{n}$ of $K^{*}$, we define $A_{1} \cdots A_{n}$ similarly. We also set $A^{-1}:=\left\{a^{-1} \mid a \in A\right\}$. Observe that any finitedimensional k-subspace $V$ of $K$ can be realized as $V=\mathrm{k}\langle A\rangle$, where $A$ is any finite subset of nonzero vectors spanning $V$. Also, when $V_{1}$ and $V_{2}$ are two k-vector spaces in $K, V_{1} V_{2} \subseteq \mathrm{k}\left\langle V_{1} V_{2}\right\rangle$ but $V_{1} V_{2}$ is not a vector space in general.

In what follows we aim to give some estimates of $\operatorname{dim}_{\mathrm{k}}(A B)$ or more generally of $\operatorname{dim}_{\mathrm{k}}\left(A_{1} \cdots A_{r}\right)$ where $A_{1}, \ldots, A_{r}$ are finite subsets of $K^{*}$. The following is straightforward

$$
\max \left(\operatorname{dim}_{\mathrm{k}}(A), \operatorname{dim}_{\mathrm{k}}(B)\right) \leq \operatorname{dim}_{\mathrm{k}}(A B) \leq \operatorname{dim}_{\mathrm{k}}(A) \operatorname{dim}_{\mathrm{k}}(B) .
$$

The methods we will use to estimate $\operatorname{dim}_{\mathrm{k}}(A B)$ are quite analogous to the tools used to estimate the cardinality $|A B|$ of the product set $A B$ where $A$ and $B$ are subsets of a given group. Many 
results on estimates of product sets have a linear analogue for the dimension of the space generated by such products. Nevertheless, there are crucial differences due notably to the fact that $\mathrm{k}\langle A\rangle$ and $\mathrm{k}\left\langle A^{-1}\right\rangle$ have not the same dimension in general, whereas $A$ and $A^{-1}$ has the same cardinality. This can be easily verified by taking $\mathrm{k}=\mathbb{C}, K=\mathbb{C}(t)$ the function field in the indeterminate $t$ and $A_{n}:=\{(t-a) \mid a=1, \ldots, n\}$. Indeed, $\operatorname{dim}_{\mathbb{C}}\left(A_{n}\right)=2$, but $\operatorname{dim}_{\mathbb{C}}\left(A_{n}^{-1}\right)=n$. Also observe that a finite abelian group has only a finite number of subgroups, whereas a finite commutative extension of a commutative field have an infinite number of intermediate extensions when it is not separable.

The two following elementary lemmas will be useful.

Lemma 2.1 Let $A$ be a finite subset of $K^{*}$ such that $\mathrm{k}\left\langle A^{2}\right\rangle=\mathrm{k}\langle A\rangle$. Then $\mathrm{k}\langle A\rangle$ is a division ring.

Proof. Observe that $\mathrm{k}\left\langle A^{2}\right\rangle=\mathrm{k}\langle A\rangle$ means that $\mathrm{k}\langle A\rangle$ is closed under multiplication. Then, for any nonzero $a \in \mathrm{k}\langle A\rangle$, the map $\varphi_{a}: \mathrm{k}\langle A\rangle \rightarrow \mathrm{k}\langle A\rangle$ which sends $\alpha \in \mathrm{k}\langle A\rangle$ on $\varphi_{a}(\alpha)=a \alpha$ is a k-linear automorphism of the space $\mathrm{k}\langle A\rangle$. In particular, $\varphi_{a}$ is surjective. Since $a \in \mathrm{k}\langle A\rangle, 1$ belongs to $\mathrm{k}\langle A\rangle$. Now since $1 \in \mathrm{k}\langle A\rangle, \alpha=a^{-1} \in A$ and $\mathrm{k}\langle A\rangle$ is a field.

Lemma 2.2 Consider a finite-dimensional k-subspace $V$ of $K$ and a sub division ring $H$ of $K$ containing $\mathrm{k}$ such that $H V=V$. Then there exists a finite subset $S$ of $K^{*}$ such that

$$
V=\bigoplus_{s \in S} H s
$$

In particular, $V$ is a left $H$-module of dimension $|S| \operatorname{dim}_{\mathrm{k}}(H)$, and (1) gives its decomposition into irreducible components.

Proof. For any nonzero vector $v$ in $V, H v$ is a k-subspace of $V$. In particular $\operatorname{dim}_{\mathrm{k}}(H v)=$ $\operatorname{dim}_{\mathrm{k}}(H)$ is finite. Moreover if $s$ is a nonzero vector in $V \backslash\left(H s_{1} \oplus \cdots \oplus H s_{t}\right)$, we have $H s \cap\left(H s_{1} \oplus\right.$ $\left.\cdots \oplus H s_{t}\right)=\{0\}$. The lemma easily follows.

\section{Remarks:}

1. From the previous lemma applied to $V=K$, we deduce that $\operatorname{dim}_{\mathrm{k}}(H) \operatorname{divides} \operatorname{dim}_{\mathrm{k}}(K)$ when $\operatorname{dim}_{\mathrm{k}}(K)$ is finite.

2. When $V H=V$, we similarly obtain a decomposition into right $H$-modules $V=\bigoplus_{s \in S} s H$.

For any subset $X$ in $K^{*}$, we set

$$
H_{\mathrm{k}, l}(X):=\{h \in K \mid h \mathrm{k}\langle X\rangle \subseteq \mathrm{k}\langle X\rangle\} \text { and } H_{\mathrm{k}, r}(X):=\{h \in K \mid \mathrm{k}\langle X\rangle h \subseteq \mathrm{k}\langle X\rangle\}
$$

for the left and right stabilizers of $\mathrm{k}\langle X\rangle$ in $K$. Clearly $H_{\mathrm{k}, l}(X)$ and $H_{\mathrm{k}, r}(X)$ are division rings containing k. In particular, when $K$ is a field, $H_{\mathrm{k}, l}(X)=H_{\mathrm{k}, r}(X)$ is a commutative extension of $\mathrm{k}$ that we simply write as $H_{\mathrm{k}}(X)$. If $H_{\mathrm{k}, l}(X)$ (resp. $\left.H_{\mathrm{k}, r}(X)\right)$ is not equal to $\mathrm{k}$, we says that $\mathrm{k}\langle X\rangle$ is left periodic (resp. right periodic). When $\mathrm{k}\langle X\rangle$ is finite-dimensional, there exists by Lemma $2.2 \mathrm{a}$ finite subset $S$ in $\mathrm{k}\langle X\rangle$ such that $\mathrm{k}\langle X\rangle=\oplus_{s \in S} H_{\mathrm{k}, l}(X) s$ (resp. $\left.\mathrm{k}\langle X\rangle=\oplus_{s \in S} s H_{\mathrm{k}, r}(X)\right)$. Observe also for $X$ and $Y$ two finite subsets of $K$, if $\langle X\rangle$ is left periodic (resp. $\langle Y\rangle$ is right periodic), then $\langle X Y\rangle$ is left periodic (resp. right periodic). Indeed, for $\langle X\rangle$ left periodic, we have $H_{\mathrm{k}, l}(X) \neq \mathrm{k}$ and $H_{\mathrm{k}, l}(X)\langle X\rangle \subseteq\langle X\rangle$. By linearity of the multiplication in $K$, this gives $H_{\mathrm{k}, l}(X)\langle X Y\rangle \subseteq\langle X Y\rangle$ thus $H_{\mathrm{k}, l}(X) \subseteq H_{\mathrm{k}, l}(X Y)$ and $H_{\mathrm{k}, l}(X Y) \neq \mathrm{k}$. The case $\langle Y\rangle$ right periodic is similar. 


\subsection{Product sets in abelian group and linear setting}

As observed in [4], estimates for cardinality of product sets in abelian groups can be obtained from their linear counterparts in fields. To do this consider an abelian group $G$ and assume

$$
G \simeq \mathbb{Z}^{l} \times \mathbb{Z} / t_{1} \mathbb{Z} \times \cdots \times \mathbb{Z} / t_{r} \mathbb{Z}
$$

The idea is to construct a field extension $K$ of $\mathrm{k}=\mathbb{C}$ such that the multiplicative group of $K$ contains a subgroup isomorphic to $G$ whose elements are linearly independent over $\mathbb{C}$. Consider first the field $E=\mathbb{C}\left(x_{1}, \ldots, x_{r}\right)$ of rational functions over the indeterminates $x_{1}, \ldots, x_{r}\left(x_{1}, \ldots, x_{r}\right.$ are thus assumed algebraically independent). Let $F$ be the decomposition field of the polynomial

$$
P(Z)=\left(Z-x_{1}^{t_{1}}\right) \cdots\left(Z-x_{r}^{t_{r}}\right) \in E[Z]
$$

over $E$. There exist elements $\sqrt[t_{1}]{x_{1}}, \ldots, \sqrt[t_{r}]{x_{r}}$ in $F$ such that $F=E\left[\sqrt[t_{1}]{x_{1}}, \ldots, \sqrt[t_{r}]{x_{r}}\right]$. Now set $K=$ $F\left(T_{1}, \ldots, T_{l}\right)$ the field of rational functions over $E$ in the algebraically independent indeterminates $T_{1}, \ldots, T_{l}$.

There exists in $G$ non torsion elements $u_{1}, \ldots, u_{l}$ and elements $\gamma_{1}, \ldots, \gamma_{r}$ respectively of order $t_{1}, \ldots, t_{r}$ such that each element $g \in G$ can be written uniquely on the form $g=u_{1}^{a_{1}} \cdots u_{l}^{a_{l}} \gamma_{1}^{b_{1}} \cdots \gamma_{r}^{b_{r}}$ where $a_{i} \in \mathbb{Z}$ for any $i=1, \ldots, l$ and $b_{j} \in\left\{0, \ldots, t_{j}-1\right\}$ for any $j=1, \ldots, r$. One associates to $g \in G$ the element $\eta(g) \in K \backslash\{0\}$ such that

$$
\eta(g)=T_{1}^{a_{1}} \cdots T_{l}^{a_{l}}\left(\sqrt[t_{1}]{x_{1}}\right)^{b_{1}} \cdots\left(\sqrt[t_{r}]{x_{r}}\right)^{b_{r}} .
$$

Clearly $\eta$ is a group embedding from $G$ to the multiplicative group of $K$ and the elements of $\eta(G)$ are linearly independent over $\mathbb{C}$ in $K$. Consider a finite subset $X \subseteq G$. Let $\Phi(X)=\mathbb{C}\langle\eta(X)\rangle$. We also define

$$
S(X)=\{g \in G \mid g X=X\} \text { and } H_{\mathbb{C}}(X)=\{x \in K \mid x \Phi(X) \subseteq \Phi(X)\} .
$$

Observe that $S(X)$ is a finite subgroup of $X$ and $H_{\mathbb{C}}(X) \subseteq K$ is a field extension of $\mathbb{C}$.

We refer to [4] for the proof of the following proposition (which uses Galois correspondence for Assertion 3). This proposition provides a correspondence between cardinality and dimension which allows one to recover classical results from their dimensional counterparts. This applies for instance to Theorem 2.7, or Theorem 3.3.'

Proposition 2.3 Given $X, Y$ two finite subsets in an abelian $G$, we have

1. $|X|=\operatorname{dim}_{\mathbb{C}}(\Phi(X))$,

2. $|X Y|=\operatorname{dim}_{\mathbb{C}}(\Phi(X) \Phi(Y))$,

3. $\Phi(S(X))=H_{\mathbb{C}}(X)$.

\subsection{Product sets in nonabelian groups and linear setting}

It would be desirable to have an analogue of Proposition 2.3 in the non abelian setting. Unfortunately, the construction of the previous paragraph can not be generalized. Indeed, there are finite subgroups which cannot be embedded in the group of invertible of a division ring (see [1]). 
In this paragraph, we will focus on a weaker connection between cardinality of product sets in a (possible non abelian) group $G$ and dimension of subspaces in the group algebra $\mathbb{C}[G]$.

Consider $G$ a (possible non abelian) group. Recall that the group algebra $\mathbb{C}[G]$ is the associative algebra generated over $\mathbb{C}$ by the elements $e_{g}, g \in G$ and the relations $e_{g} e_{g^{\prime}}=e_{g g^{\prime}}$ for any $g, g^{\prime} \in G$. To any finite subset $X$ of $G$ we associate the $\mathbb{C}$-subspace $\mathrm{k}\left\langle A_{X}\right\rangle$ of $\mathbb{C}[G]$ where $A_{X}=\left\{e_{g} \mid g \in\right.$ $X\} \subset \mathbb{C}[G]$.

Given two finite subspaces $X$ and $Y$ of $\mathbb{C}[G]$, we have clearly

$$
|X|=\operatorname{dim}_{\mathbb{C}}\left(A_{X}\right) \text { and }|X Y|=\operatorname{dim}_{\mathbb{C}}\left(A_{X} A_{Y}\right) .
$$

In Section 4, we will see that it is possible to recover some estimates for the cardinality of product sets in groups from analogous statements in ring algebras.

\subsection{The linear Kneser theorem}

We now recall the linear Kneser theorem stated in [4] for fields.

Theorem 2.4 Let $K$ be a commutative extension of $\mathrm{k}$. Assume every algebraic element in $K$ is separable over k. Let $A$ and $B$ be two nonempty, finite subsets of $K^{*}$. Then

$$
\operatorname{dim}_{\mathrm{k}}(A B) \geq \operatorname{dim}_{\mathrm{k}}(A)+\operatorname{dim}_{\mathrm{k}}(B)-\operatorname{dim}_{\mathrm{k}}\left(H_{\mathrm{k}}(A B)\right) .
$$

In $\S$ [5.3, we will give a noncommutative version of the following corollary due to Tao [18] where the separability hypothesis can be relaxed.

Corollary 2.5 Let $K$ be a commutative extension of $\mathrm{k}$. Assume every algebraic element in $K$ is separable over $\mathrm{k}$. Let $A$ be a nonempty, finite subset of $K^{*}$ such that $\operatorname{dim}_{\mathrm{k}}\left(A^{2}\right) \leq(2-\varepsilon) \operatorname{dim}_{\mathrm{k}}(A)$ for a real $\varepsilon$ with $0<\varepsilon \leq 1$. Then there exists a field $H$ that is finite-dimensional over $\mathrm{k}$ and $a$ finite non empty, subset $X$ of $K^{*}$ with $|X| \leq \frac{2}{\varepsilon}-1$ such that $\mathrm{k}\left\langle A^{2}\right\rangle \subseteq \bigoplus_{x \in X} x H$.

Proof. By the previous theorem, we must have $\operatorname{dim}_{\mathrm{k}}\left(H_{\mathrm{k}}\left(A^{2}\right)\right) \geq 2 \operatorname{dim}_{\mathrm{k}}(A)-\operatorname{dim}_{\mathrm{k}}\left(A^{2}\right) \geq$ $\varepsilon \operatorname{dim}_{\mathrm{k}}(A)$. By Lemma 2.2, there exists a finite subset $X$ of $K^{*}$ such that $\mathrm{k}\left\langle A^{2}\right\rangle=\bigoplus_{x \in X} x H$. We thus have

$$
|X|=\frac{\operatorname{dim}_{\mathrm{k}}\left(A^{2}\right)}{\operatorname{dim}_{\mathrm{k}}(H)} \leq \frac{(2-\varepsilon) \operatorname{dim}_{\mathrm{k}}(A)}{\varepsilon \operatorname{dim}_{\mathrm{k}}(A)} \leq \frac{2}{\varepsilon}-1
$$

as desired.

\section{Remarks:}

1. Theorem 2.4 can be regarded as a linear version of Kneser's theorem. Recall that this theorem establishes that for any nonempty, finite subsets $A$ and $B$ in an abelian group $G$ (written multiplicatively), we have $|A B| \geq|A|+|B|-|H|$, where $H$ is the stabilizer of $A B$ in $G$.

2. As proved in [4, the linear Kneser theorem implies Kneser's theorem. This follows from Proposition 2.3.

3. The separability hypothesis is crucial in the proof of the theorem as the finite extensions of $\mathrm{k}$ should have a finite number of intermediate extensions. Nevertheless, it is conjectured in [9] that the separability hypothesis can be relaxed. Also observe that the separability hypothesis is always satisfied in characteristic zero. 
Like the original Kneser theorem, Theorem 2.4 can be generalized for Minkowski products of any finite number of finite subsets of $K^{*}$. The following theorem is not explicitly stated in [4]. We give its proof below for completion. We first need the following easy lemma.

Lemma 2.6 Let $K$ be a commutative extension of $\mathrm{k}$. Consider an integer $n \geq 2$ and a collection of finite, nonempty subsets $A_{1}, \ldots, A_{n}$ of $K^{*}$ such that

$$
\operatorname{dim}_{\mathrm{k}}\left(\prod_{i=1}^{j} A_{i}\right) \geq \operatorname{dim}_{\mathrm{k}}\left(\prod_{i=1}^{j-1} A_{i}\right)+\operatorname{dim}_{\mathrm{k}}\left(A_{j}\right)-1
$$

holds for $j=2, \ldots, n$. Then

$$
\operatorname{dim}_{\mathrm{k}}\left(\prod_{i=1}^{n} A_{i}\right) \geq \sum_{i=1}^{n} \operatorname{dim}_{\mathrm{k}}\left(A_{i}\right)-n+1 .
$$

Proof. We proceed by induction on $j$. For $j=2$, we have $\operatorname{dim}_{\mathrm{k}}\left(A_{1} A_{2}\right) \geq \operatorname{dim}_{\mathrm{k}}\left(A_{1}\right)+\operatorname{dim}_{\mathrm{k}}\left(A_{2}\right)-$ 1 by (2). Assume we have

$$
\operatorname{dim}_{\mathrm{k}}\left(\prod_{i=1}^{j} A_{i}\right) \geq \sum_{i=1}^{j} \operatorname{dim}_{\mathrm{k}}\left(A_{i}\right)-j+1
$$

Writing (21) with $j+1$ gives

$$
\operatorname{dim}_{\mathrm{k}}\left(\prod_{i=1}^{j+1} A_{i}\right) \geq \operatorname{dim}_{\mathrm{k}}\left(\prod_{i=1}^{j} A_{i}\right)+\operatorname{dim}_{\mathrm{k}}\left(A_{j+1}\right)-1 .
$$

Combining with (3), one obtains

$$
\operatorname{dim}_{\mathrm{k}}\left(\prod_{i=1}^{j+1} A_{i}\right) \geq \sum_{i=1}^{j} \operatorname{dim}_{\mathrm{k}}\left(A_{i}\right)-j+\operatorname{dim}_{\mathrm{k}}\left(A_{j+1}\right)
$$

as desired.

Theorem 2.7 Let $K$ be a commutative extension of $\mathrm{k}$. Consider a collection of finite, nonempty subsets $A_{1}, \ldots, A_{n}$ of $K^{*}$ Set $H:=H_{\mathrm{k}}\left(A_{1} \cdots A_{n}\right)$. The following statements are equivalent:

1. $\operatorname{dim}_{\mathrm{k}}\left(A_{1} \cdots A_{n}\right) \geq \sum_{i=1}^{n} \operatorname{dim}_{\mathrm{k}}\left(A_{i} H\right)-(n-1) \operatorname{dim}_{\mathrm{k}}(H)$,

2. $\operatorname{dim}_{\mathrm{k}}\left(A_{1} \cdots A_{n}\right) \geq \sum_{i=1}^{n} \operatorname{dim}_{\mathrm{k}}\left(A_{i}\right)-(n-1) \operatorname{dim}_{\mathrm{k}}(H)$,

3. either $\operatorname{dim}_{\mathrm{k}}\left(A_{1} \cdots A_{n}\right) \geq \sum_{i=1}^{n} \operatorname{dim}_{\mathrm{k}}\left(A_{i}\right)-(n-1)$ or $\mathrm{k}\left\langle A_{1} \cdots A_{n}\right\rangle$ is periodic,

4. any one of the above three statements in the case $n=2$.

Proof. (I): We obtain $1 \Rightarrow 2$ by using $\operatorname{dim}_{\mathrm{k}}\left(A_{i} H\right) \geq \operatorname{dim}_{\mathrm{k}}\left(A_{i}\right)$ for any $i=1, \ldots, n$. The implication $2 \Longrightarrow 3$ is immediate. To prove $3 \Longrightarrow 1$, we first observe the implication is true when $H=\mathrm{k}$. Note that $H\left(A_{1} \cdots A_{n}\right)$ is, by definition, not periodic. When $\mathrm{k}\left\langle A_{1} \cdots A_{n}\right\rangle$ is periodic using the base field $\mathrm{k}$, it is not periodic using the base field $H$. We thus can apply assertion 3 by 
considering $K$ as a commutative extension of $H$. This gives $\operatorname{dim}_{H}\left(A_{1} \cdots A_{n}\right) \geq \sum_{i=1}^{n} \operatorname{dim}_{H}\left(A_{i}\right)-$ $(n-1)$. Multiplying this inequality by $\operatorname{dim}_{\mathrm{k}}(H)$ yields

$$
\operatorname{dim}_{H}\left(A_{1} \cdots A_{n}\right) \operatorname{dim}_{\mathrm{k}}(H) \geq \sum_{i=1}^{n} \operatorname{dim}_{H}\left(A_{i}\right) \operatorname{dim}_{\mathrm{k}}(H)-(n-1) \operatorname{dim}_{\mathrm{k}}(H)
$$

which is equivalent to 1 since $\operatorname{dim}_{H}\left(A_{1} \cdots A_{n}\right) \operatorname{dim}_{\mathrm{k}}(H)=\operatorname{dim}_{\mathrm{k}}\left(A_{1} \cdots A_{n}\right)$ and $\operatorname{dim}_{H}\left(A_{i}\right) \operatorname{dim}_{\mathrm{k}}(H)=$ $\operatorname{dim}_{\mathrm{k}}\left(A_{i} H\right)$ for any $i=1, \ldots, n$.

(II): It remains to prove that assertion 3 is equivalent to the following:

3' Given two finite, nonempty subsets $A$ and $B$ of $K^{*}$, either $\operatorname{dim}_{\mathrm{k}}(A B) \geq \operatorname{dim}_{\mathrm{k}}(A)+\operatorname{dim}_{\mathrm{k}}(B)-1$ or $\mathrm{k}\langle A B\rangle$ is periodic.

Clearly $3 \Longrightarrow 3^{\prime}$. Now assume $3^{\prime}$ holds and $\operatorname{dim}_{\mathrm{k}}\left(A_{1} \cdots A_{n}\right)<\sum_{i=1}^{n} \operatorname{dim}_{\mathrm{k}}\left(A_{i}\right)-(n-1)$. Then, by Lemma 2.6, there exists $j \in\{2, \ldots, n\}$ such that

$$
\operatorname{dim}_{\mathrm{k}}\left(\prod_{i=1}^{j} A_{i}\right)<\operatorname{dim}_{\mathrm{k}}\left(\prod_{i=1}^{j-1} A_{i}\right)+\operatorname{dim}_{\mathrm{k}}\left(A_{j}\right)-1
$$

By applying $3^{\prime}$ with $A=\prod_{i=1}^{j-1} A_{i}$ and $B=A_{j}$, we obtain that $\mathrm{k}\langle A B\rangle=\mathrm{k}\left\langle\prod_{i=1}^{j} A_{i}\right\rangle$ is periodic. Therefore $\mathrm{k}\left\langle\prod_{i=1}^{n} A_{i}\right\rangle$ is also periodic. This shows that $3^{\prime} \Longrightarrow 3$.

Remark: The four assertions of the theorem are equivalent without the separability hypothesis of Theorem 2.4.

\section{Plünnecke-type estimates in division rings}

Plünnecke-type estimates permit one to obtain upper bounds on the cardinality of sumsets in abelian groups as stated in Theorem 1.1. Plünnecke's result was first stated for $G=\mathbb{Z}$, but his proof, based on a graph-theoretic method, can be extended to arbitrary abelian groups. Very recently, Petridis gave a surprisingly elegant and short proof of Theorem 1.1. This proof can be adapted to the context of division rings as explained in the following paragraphs. In Section 4, we will consider the case of associative unital algebras.

\subsection{Minimal growth under multiplication}

Let $K$ be a division ring containing the field $\mathrm{k}$ in its center. Consider two finite subsets $A$ and $B$ of $K^{*}$. For any $\mathrm{k}$-subspace $V \neq\{0\}$ of $\mathrm{k}\langle A\rangle$ (hence $V$ is finite-dimensional), we set

$$
r(V):=\frac{\operatorname{dim}_{\mathrm{k}}(V B)}{\operatorname{dim}_{\mathrm{k}}(V)}
$$

for the growth of $V$ under multiplication by $B$. Write $\rho:=\min _{V \subseteq \mathrm{k}\langle A\rangle, V \neq\{0\}} r(V)$. Since the image of the map $r$ is contained in a discrete set of positive numbers, there exists a nonempty set $X \subseteq \mathrm{k}\langle A\rangle$ such that $r(\mathrm{k}\langle X\rangle)=\rho$. We thus have, $\operatorname{dim}_{\mathrm{k}}(X B)=\rho \operatorname{dim}_{\mathrm{k}}(X)$ and $\operatorname{dim}_{\mathrm{k}}(X B) / \operatorname{dim}_{\mathrm{k}}(X) \leq$ $\operatorname{dim}_{\mathrm{k}}(Z B) / \operatorname{dim}_{\mathrm{k}}(Z)$ for any $Z \subseteq \mathrm{k}\langle A\rangle$. 
Proposition 3.1 Under the previous hypotheses, we have, for any finite set $C$ in $K^{*}$,

$$
\operatorname{dim}_{\mathrm{k}}(C X B) \leq \rho \operatorname{dim}_{\mathrm{k}}(C X)=\frac{\operatorname{dim}_{\mathrm{k}}(C X) \operatorname{dim}_{\mathrm{k}}(X B)}{\operatorname{dim}_{\mathrm{k}}(X)} .
$$

Proof. Write $C=\left\{c_{1}, \ldots, c_{r}\right\}$. We construct by induction subsets $X_{1}, \ldots, X_{r}$ of $X$ such that

$$
\sum_{i=1}^{j} c_{i} \mathrm{k}\langle X\rangle=\bigoplus_{i=1}^{j} c_{i} \mathrm{k}\left\langle X_{i}\right\rangle
$$

for any $j=1, \ldots r$. Set $X_{1}=X$. Now assume we have constructed subsets $X_{1}, \ldots, X_{j-1}$ of $X$ such that

$$
\sum_{i=1}^{j-1} c_{i} \mathrm{k}\langle X\rangle=\bigoplus_{i=1}^{j-1} c_{i} \mathrm{k}\left\langle X_{i}\right\rangle
$$

We have

$$
\sum_{i=1}^{j} c_{i} \mathrm{k}\langle X\rangle=\bigoplus_{i=1}^{j-1} c_{i} \mathrm{k}\left\langle X_{i}\right\rangle+c_{j} \mathrm{k}\langle X\rangle
$$

Let $B_{j-1}$ be any basis of $\bigoplus_{i=1}^{j-1} c_{i} \mathrm{k}\left\langle X_{i}\right\rangle$. Then $B_{j-1} \cup c_{j} X$ generates $\sum_{i=1}^{j} c_{i} \mathrm{k}\langle X\rangle$. There thus exists a subset $X_{j} \subseteq X$ such that

$B_{j-1} \cup c_{j} X_{j}$ is a basis of $\sum_{i=1}^{j} c_{i} \mathrm{k}\langle X\rangle$. We then have

$$
\sum_{i=1}^{j} c_{i} \mathrm{k}\langle X\rangle=\bigoplus_{i=1}^{j} c_{i} \mathrm{k}\left\langle X_{i}\right\rangle
$$

as desired.

By construction of the sets $X_{i}$, we have

$$
\operatorname{dim}_{\mathrm{k}}\left(\sum_{i=1}^{j} c_{i} \mathrm{k}\langle X\rangle\right)=\sum_{i=1}^{j} \operatorname{dim}_{\mathrm{k}}\left(c_{i} X_{i}\right)=\sum_{i=1}^{j} \operatorname{dim}_{\mathrm{k}}\left(X_{i}\right)
$$

for any $j=1, \ldots, r$.

As in the proof of Petridis, we now proceed by induction on $r$. When $r=1, \operatorname{dim}_{\mathrm{k}}\left(c_{1} X B\right)=$ $\operatorname{dim}_{\mathrm{k}}(X B)=\rho \operatorname{dim}_{\mathrm{k}}(X)=\rho \operatorname{dim}_{\mathrm{k}}\left(c_{1} X\right)$. Assume $r>1$. Set $V_{r}:=c_{r}^{-1} \sum_{a=1}^{r-1} c_{a} \mathrm{k}\langle X\rangle \cap \mathrm{k}\langle X\rangle$ and $\mathrm{k}\langle X\rangle=\mathrm{k}\left\langle X_{r}\right\rangle \oplus V_{r}$. We then have $c_{r} V_{r} \subseteq \sum_{a=1}^{r-1} c_{a} \mathrm{k}\langle X\rangle$ and $c_{r} \mathrm{k}\left\langle V_{r} B\right\rangle \subseteq \sum_{a=1}^{r-1} c_{a} \mathrm{k}\langle X B\rangle$. Since $\mathrm{k}\left\langle V_{r} B\right\rangle$ is a subspace of $\mathrm{k}\langle X B\rangle$, this gives

$$
\mathrm{k}\langle C X B\rangle=\sum_{a=1}^{r} c_{a} \mathrm{k}\langle X B\rangle=\sum_{a=1}^{r-1} c_{a} \mathrm{k}\langle X B\rangle+c_{r} \mathrm{k}\langle X B\rangle=\sum_{a=1}^{r-1} c_{a} \mathrm{k}\langle X B\rangle+c_{r} W
$$

where $W$ is a k-subspace of $\mathrm{k}\langle X B\rangle$ such that $\mathrm{k}\langle X B\rangle=W \oplus \mathrm{k}\left\langle V_{r} B\right\rangle$. We have, in particular, $\operatorname{dim}_{\mathrm{k}}\left(c_{r} W\right)=\operatorname{dim}_{\mathrm{k}}(W)=\operatorname{dim}_{\mathrm{k}}(X B)-\operatorname{dim}_{\mathrm{k}}\left(V_{r} B\right)$. We thus obtain

$$
\operatorname{dim}_{\mathrm{k}}(C X B) \leq \operatorname{dim}_{\mathrm{k}}\left(\sum_{a=1}^{r-1} c_{a} \mathrm{k}\langle X B\rangle\right)+\operatorname{dim}_{\mathrm{k}}(X B)-\operatorname{dim}_{\mathrm{k}}\left(V_{r} B\right) .
$$


By the induction hypothesis, we have

$$
\operatorname{dim}_{\mathrm{k}}\left(\sum_{a=1}^{r-1} c_{a} \mathrm{k}\langle X B\rangle\right)=\operatorname{dim}_{\mathrm{k}}\left(C^{\prime} X B\right) \leq \rho \operatorname{dim}_{\mathrm{k}}\left(C^{\prime} X\right)=\rho \operatorname{dim}_{\mathrm{k}}\left(\sum_{a=1}^{r-1} c_{a} \mathrm{k}\langle X\rangle\right)
$$

with $C^{\prime}=C \backslash\left\{c_{r}\right\}$. Thus by using (5) with $j=r-1$, one gets

$$
\operatorname{dim}_{\mathrm{k}}\left(\sum_{a=1}^{r-1} c_{a} \mathrm{k}\langle X B\rangle\right) \leq \rho \sum_{a=1}^{r-1} \operatorname{dim}_{\mathrm{k}}\left(c_{a} X_{a}\right)
$$

Since $V_{r} \subseteq \mathrm{k}\langle X\rangle$, we have $\operatorname{dim}_{\mathrm{k}}\left(V_{r} B\right) \geq \rho \operatorname{dim}_{\mathrm{k}}\left(V_{r}\right)$. By definition of $X$, we have $\operatorname{dim}_{\mathrm{k}}(X B)=$ $\rho \operatorname{dim}_{\mathrm{k}}(X)$. Therefore

$$
\operatorname{dim}_{\mathrm{k}}(X B)-\operatorname{dim}_{\mathrm{k}}\left(V_{r} B\right) \leq \rho \operatorname{dim}_{\mathrm{k}}(X)-\rho \operatorname{dim}_{\mathrm{k}}\left(V_{r}\right) \leq \rho \operatorname{dim}_{\mathrm{k}}\left(X_{r}\right) .
$$

Combining the two previous inequalities with ([6), we finally obtain

$$
\begin{gathered}
\operatorname{dim}_{\mathrm{k}}(C X B) \leq \rho \sum_{a=1}^{r-1} \operatorname{dim}_{\mathrm{k}}\left(c_{a} X_{a}\right)+\rho \operatorname{dim}_{\mathrm{k}}\left(c_{r} X_{r}\right) \leq \\
\rho \sum_{a=1}^{r} \operatorname{dim}_{\mathrm{k}}\left(c_{a} X_{a}\right)=\rho \operatorname{dim}_{\mathrm{k}}\left(\sum_{a=1}^{r} c_{a} \mathrm{k}\langle X\rangle\right)=\rho \operatorname{dim}_{\mathrm{k}}(C X) .
\end{gathered}
$$

where the second to last equality is obtained by (5) with $j=r$.

Corollary 3.2 Let $K$ be a division ring containing the field $\mathrm{k}$ in its center. Consider two finite subsets $A$ and $B$ of $K^{*}$. Assume $\alpha$ is a positive real such that $\operatorname{dim}_{\mathrm{k}}(A B) \leq \alpha \operatorname{dim}_{\mathrm{k}}(A)$. Then there exists a subset $X \subseteq \mathrm{k}\langle A\rangle$ such that, for any finite subset $C$ of $K^{*}, \operatorname{dim}_{\mathrm{k}}(C X B) \leq \alpha \operatorname{dim}_{\mathrm{k}}(C X)$.

Proof. Let $X \subseteq \mathrm{k}\langle A\rangle$ be such that $\rho=r(X)$. We have

$$
\rho=\frac{\operatorname{dim}_{\mathrm{k}}(X B)}{\operatorname{dim}_{\mathrm{k}}(X)} \leq \frac{\operatorname{dim}_{\mathrm{k}}(A B)}{\operatorname{dim}_{\mathrm{k}}(A)} \leq \alpha
$$

by definition of $\rho$. We then apply Proposition 3.1, which yields $\operatorname{dim}_{\mathrm{k}}(C X B) \leq \alpha \operatorname{dim}_{\mathrm{k}}(C X)$.

\subsection{Plünnecke upper bounds for $\operatorname{dim}_{\mathrm{k}}(A B)$}

We assume in this paragraph that $K$ is a commutative extension of $\mathrm{k}$. The following theorem can be regarded as a linear version of Theorem 1.1. In fact, it is a linear version of the slightly stronger result obtained by Petridis where $X$ is the same for any positive integer $n$.

Theorem 3.3 Let $A$ and $B$ be nonempty, finite subsets in $K^{*}$. Assume that $\operatorname{dim}_{\mathrm{k}}(A B) \leq \alpha \operatorname{dim}_{\mathrm{k}}(A)$ where $\alpha$ is a positive real. Then there exists a subset $X \subseteq \mathrm{k}\langle A\rangle$ such that, for any positive integer $n$,

$$
\operatorname{dim}_{\mathrm{k}}\left(X B^{n}\right) \leq \alpha^{n} \operatorname{dim}_{\mathrm{k}}(X) .
$$

In particular, $\operatorname{dim}_{\mathrm{k}}\left(A^{2}\right) \leq \alpha \operatorname{dim}_{\mathrm{k}}(A)$ implies that $\operatorname{dim}\left(A^{n}\right) \leq \alpha^{n} \operatorname{dim}(A)$. 
Proof. The proof is by induction on $n$. Let $X$ be such that $\rho=r(X)$. For $n=1$, we have

$$
\operatorname{dim}_{\mathrm{k}}(X B)=\operatorname{dim}_{\mathrm{k}}(X) \rho \leq \operatorname{dim}_{\mathrm{k}}(X) \frac{\operatorname{dim}_{\mathrm{k}}(A B)}{\operatorname{dim}_{\mathrm{k}}(A)} \leq \alpha \operatorname{dim}_{\mathrm{k}}(X)
$$

For any $n>1$, we set $C=B^{n-1}$. By applying Proposition 3.1 with $C=B^{n-1}$, we have

$$
\operatorname{dim}_{\mathrm{k}}\left(X B^{n}\right)=\operatorname{dim}_{\mathrm{k}}\left(B^{n-1} X B\right) \leq \frac{\operatorname{dim}_{\mathrm{k}}\left(B^{n-1} X\right) \operatorname{dim}_{\mathrm{k}}(X B)}{\operatorname{dim}_{\mathrm{k}}(X)} .
$$

Next, by the induction hypothesis, $\operatorname{dim}_{\mathrm{k}}\left(B^{n-1} X\right)=\operatorname{dim}_{\mathrm{k}}\left(X B^{n-1}\right) \leq \alpha^{n-1} \operatorname{dim}(X)$. Therefore

$$
\operatorname{dim}_{\mathrm{k}}\left(X B^{n}\right) \leq \alpha^{n-1} \operatorname{dim}_{\mathrm{k}}(X B) \leq \alpha^{n} \operatorname{dim}_{\mathrm{k}}(X)
$$

where the last inequality follows from the case $n=1$.

\section{Remarks:}

1. Observe that commutativity is crucial in the previous proof. Nevertheless, the conclusion of Theorem 3.3 remains valid in a division ring with the additional assumption

$$
a b=b a \text { for any } a \in A \text { and } b \in B \text {. }
$$

Indeed, we then have $x y=y x$ for any $x \in \mathrm{k}\langle A\rangle$ and $y \in \mathrm{k}\langle B\rangle$. So in the previous proof we still have $\mathrm{k}\left\langle X B^{n}\right\rangle=\mathrm{k}\left\langle B^{n-1} X B\right\rangle$ and $\mathrm{k}\left\langle B^{n-1} X\right\rangle=\mathrm{k}\left\langle X B^{n-1}\right\rangle$ since $X \subset \mathrm{k}\langle A\rangle$.

2. Assertions 1 and 2 of Proposition 2.3 permits one to recover the Plünnecke-Ruzsa cardinality estimates for abelian groups.

\subsection{Double and triple product}

We now show how to estimate, in a field $K$, the dimension of the vector span generated by a triple products set in terms of the dimensions of the vector spans of the corresponding double product set. This is a linear version of Theorem 1.9.2 in [17] :

Theorem 3.4 Let $A, B$ and $C$ be finite subsets of a group $G$. Then

$$
|A B C|^{2} \leq|A B||B C| \max _{b \in B}|A b C| .
$$

Theorem 3.5 Consider a division ring $K$ containing the field $\mathrm{k}$ in its center. Let $A, B$ and $C$ be finite, nonempty subsets of $K^{*}$. Then

$$
\left(\operatorname{dim}_{\mathrm{k}}(A B C)\right)^{2} \leq \operatorname{dim}_{\mathrm{k}}(A B) \operatorname{dim}_{\mathrm{k}}(B C) \max _{b \in B}\left\{\operatorname{dim}_{\mathrm{k}}(A b C)\right\} .
$$

In particular, when $K$ is a field, we have

$$
\operatorname{dim}_{\mathrm{k}}(A B C)^{2} \leq \operatorname{dim}_{\mathrm{k}}(A B) \operatorname{dim}_{\mathrm{k}}(B C) \operatorname{dim}_{\mathrm{k}}(A C) .
$$


Proof. We proceed by induction on $|B|$. When $B=\{b\}$, we obtain

$$
\operatorname{dim}_{\mathrm{k}}(A b C)^{2} \leq \operatorname{dim}_{\mathrm{k}}(A b) \operatorname{dim}_{\mathrm{k}}(b C) \operatorname{dim}_{\mathrm{k}}(A b C)
$$

by observing that $\operatorname{dim}_{\mathrm{k}}(A b C) \leq \operatorname{dim}_{\mathrm{k}}(A b) \operatorname{dim}_{\mathrm{k}}(C)$ and $\operatorname{dim}_{\mathrm{k}}(C)=\operatorname{dim}_{\mathrm{k}}(b C)$. Now assume $|B|>1$ and fix $b \in B$ such that $\max _{u \in B}\left\{\operatorname{dim}_{\mathrm{k}}(A u C)\right\}=\operatorname{dim}_{\mathrm{k}}(A b C)$. Set $m=\operatorname{dim}_{\mathrm{k}}(A b C)$. Write $B^{\prime}=$ $B \backslash\{b\}$. Set $A=\left\{a_{1}, \ldots, a_{r}\right\}$ and $C=\left\{c_{1}, \ldots, c_{s}\right\}$. We have $\mathrm{k}\langle A B\rangle=\mathrm{k}\left\langle A B^{\prime}\right\rangle+\sum_{a \in A} \mathrm{k}\langle a b\rangle$. Let $S_{A B^{\prime}}$ be a basis of $\mathrm{k}\left\langle A B^{\prime}\right\rangle$. Since $S_{A^{\prime} B} \cup A b$ generates $\mathrm{k}\langle A B\rangle$, there exists a subset $A^{\mathrm{b}}$ of $A$ such that

$$
\mathrm{k}\langle A B\rangle=\mathrm{k}\left\langle A B^{\prime}\right\rangle \oplus \bigoplus_{a \in A^{b}} \mathrm{k}\langle a b\rangle .
$$

Similarly, there exists a subset $C^{b}$ of $C$ such that

$$
\mathrm{k}\langle B C)=\mathrm{k}\left\langle B^{\prime} C\right\rangle \oplus \bigoplus_{c \in C^{b}} \mathrm{k}\langle b c\rangle .
$$

We get

$$
\begin{aligned}
\mathrm{k}\langle A B C\rangle=\mathrm{k}\left\langle A B^{\prime} C\right\rangle+\sum_{a \in A^{b}} \mathrm{k}\langle a b C\rangle=\mathrm{k}\left\langle A B^{\prime} C\right\rangle+ & \sum_{a \in A^{b}} \mathrm{k}\langle a B C\rangle= \\
& \mathrm{k}\left\langle A B^{\prime} C\right\rangle+\sum_{a \in A^{b}} \mathrm{k}\left\langle a B^{\prime} C\right\rangle+\sum_{a \in A^{b}} \sum_{c \in C^{b}} \mathrm{k}\langle a b c\rangle .
\end{aligned}
$$

But $\sum_{a \in A^{b}} \mathrm{k}\left\langle a B^{\prime} C\right\rangle \subseteq \mathrm{k}\left\langle A B^{\prime} C\right\rangle$, and thus

$$
\mathrm{k}\langle A B C\rangle=\mathrm{k}\left\langle A B^{\prime} C\right\rangle+\sum_{a \in A^{b}} \sum_{c \in C^{b}} \mathrm{k}\langle a b c\rangle
$$

Let $S_{A B^{\prime} C}$ be a basis of $\mathrm{k}\left\langle A B^{\prime} C\right\rangle$. By the previous decomposition, there exists $X \subseteq A^{b} \times B^{b}$ such that

$$
\mathrm{k}\langle A B C\rangle=\mathrm{k}\left\langle A B^{\prime} C\right\rangle \oplus \bigoplus_{(a, c) \in X} \mathrm{k}\langle a b c\rangle
$$

Set $\alpha=|X|, \beta=\left|A^{b}\right|$ and $\gamma=\left|C^{b}\right|$. We have to prove (7), that is,

$$
\left(\operatorname{dim}_{\mathrm{k}}\left(A B^{\prime} C\right)+\alpha\right)^{2} \leq\left(\operatorname{dim}_{\mathrm{k}}\left(A B^{\prime}\right)+\beta\right)\left(\operatorname{dim}_{\mathrm{k}}\left(B^{\prime} C\right)+\gamma\right) m .
$$

By the induction hypothesis, we have

$$
\operatorname{dim}_{\mathrm{k}}\left(A B^{\prime} C\right)^{2} \leq \operatorname{dim}_{\mathrm{k}}\left(A B^{\prime}\right) \operatorname{dim}_{\mathrm{k}}\left(B^{\prime} C\right) m
$$

because $\max _{u \in B^{\prime}}\left\{\operatorname{dim}_{\mathrm{k}}(A u C)\right\} \leq \max _{u \in B}\left\{\operatorname{dim}_{\mathrm{k}}(A u C)\right\}=m$. We have $\bigoplus_{(a, c) \in X} \mathrm{k}\langle a b c\rangle \subseteq \mathrm{k}\langle A b C\rangle$. So $\alpha \leq m$. Since $X \subseteq A^{b} \times B^{b}$, we have also $\alpha \leq \beta \gamma$. We get $\alpha^{2} \leq m \beta \gamma$. By multiplying in (9), this gives

$$
\alpha^{2} \operatorname{dim}_{\mathrm{k}}\left(A B^{\prime} C\right)^{2} \leq \beta \gamma \operatorname{dim}_{\mathrm{k}}\left(A B^{\prime}\right) \operatorname{dim}_{\mathrm{k}}\left(B^{\prime} C\right) m^{2} .
$$

Therefore

$$
\alpha \operatorname{dim}_{\mathrm{k}}\left(A B^{\prime} C\right) \leq m \sqrt{\beta \gamma \operatorname{dim}_{\mathrm{k}}\left(A B^{\prime}\right) \operatorname{dim}_{\mathrm{k}}\left(B^{\prime} C\right)} \leq m \frac{\gamma \operatorname{dim}_{\mathrm{k}}\left(A B^{\prime}\right)+\beta \operatorname{dim}_{\mathrm{k}}\left(B^{\prime} C\right)}{2}
$$


by applying the geometric-arithmetic means inequality. So

$$
2 \alpha \operatorname{dim}_{\mathrm{k}}\left(A B^{\prime} C\right) \leq m\left(\gamma \operatorname{dim}_{\mathrm{k}}\left(A B^{\prime}\right)+\beta \operatorname{dim}_{\mathrm{k}}\left(B^{\prime} C\right)\right) .
$$

Combining this last equality with $\alpha^{2} \leq m \beta \gamma$ and (9), we finally get

$\operatorname{dim}_{\mathrm{k}}\left(A B^{\prime} C\right)^{2}+2 \alpha \operatorname{dim}_{\mathrm{k}}\left(A B^{\prime} C\right)+\alpha^{2} \leq m\left(\operatorname{dim}_{\mathrm{k}}\left(A B^{\prime}\right) \operatorname{dim}_{\mathrm{k}}\left(B^{\prime} C\right)+\gamma \operatorname{dim}_{\mathrm{k}}\left(A B^{\prime}\right)+\beta \operatorname{dim}_{\mathrm{k}}\left(B^{\prime} C\right)+\beta \gamma\right)$

as desired.

By using Theorems 3.3 and 3.5 , we can obtain a bound for $\operatorname{dim}_{\mathrm{k}}\left(A^{3}\right) \operatorname{knowing} \operatorname{dim}_{\mathrm{k}}\left(A^{2}\right)$ and $\operatorname{dim}_{\mathrm{k}}(A)$.

Corollary 3.6 Consider a field extension $K$ of $\mathrm{k}$ and $A$ a nonempty, finite subset of $K^{*}$. Assume $\operatorname{dim}_{\mathrm{k}}(A)=m$ and $\operatorname{dim}_{\mathrm{k}}\left(A^{2}\right)=n$. Then,

$$
\operatorname{dim}_{\mathrm{k}}\left(A^{3}\right) \leq \min \left(n^{3 / 2}, \frac{n^{3}}{m^{2}}\right) .
$$

\section{Plünnecke-type estimates in associative algebras}

The arguments we have used in the proofs of Section 3 to obtain Plünnecke-type estimates in division rings in fact remain valid in the more general context of associative unital algebras with suitable hypotheses on the subspaces considered. More precisely, let $\mathcal{A}$ be a unital associative algebra over the field k. Write $U(\mathcal{A})$ for the group of invertible elements in $\mathcal{A}$. As classical examples, we can consider any matrix algebra containing the identity matrix or the group algebras.

Given a subset $A$ of $\mathcal{A}$ and $x \in \mathcal{A}, \operatorname{dim}_{\mathrm{k}}(x A)$ and $\operatorname{dim}_{\mathrm{k}}(A x)$ do not necessarily coincide with $\operatorname{dim}_{\mathrm{k}}(A)$. This is nevertheless true when $x \in U(\mathcal{A})$. Let $A, B$ and $C$ be nonempty, finite subsets of $\mathcal{A}$ such that

- $B \cap U(\mathcal{A}) \neq \emptyset$,

- $C \subseteq U(\mathcal{A})$.

Then for any k-subspace $V \neq\{0\}$ of $\mathrm{k}\langle A\rangle$,

$$
\operatorname{dim}_{\mathrm{k}}(V B) \geq \operatorname{dim}_{\mathrm{k}}(V), \quad r(V)=\frac{\operatorname{dim}_{\mathrm{k}}(V B)}{\operatorname{dim}_{\mathrm{k}}(V)}>0 \text { and } \rho:=\min _{V \subseteq \mathrm{k}\langle A\rangle, V \neq\{0\}} r(V)>0 .
$$

There thus also exists a nonempty set $\mathrm{k}\langle X\rangle \subseteq A$ such that $r(\mathrm{k}\langle X\rangle)=\rho$. One then easily verifies that the arguments used in the proof of Proposition 3.1 remain valid for the algebra $\mathcal{A}$ with the previous assumptions on $A, B$ and $C$. We then obtain the following statements which generalize Corollary 3.2 , Theorem 3.3 and Theorem 3.5 .

Corollary 4.1 Consider $A$ and $B$ two finite subsets of $\mathcal{A}$ with $B \cap U(\mathcal{A}) \neq \emptyset$. Assume $\alpha$ is a positive real such that $\operatorname{dim}_{\mathrm{k}}(A B) \leq \alpha \operatorname{dim}_{\mathrm{k}}(A)$. Then there exists a subset $X \subseteq \mathrm{k}\langle A\rangle$ such that, for any finite subset $C$ of $U(\mathcal{A}), \operatorname{dim}_{\mathrm{k}}(C X B) \leq \alpha \operatorname{dim}_{\mathrm{k}}(C X)$. 
Theorem 4.2 Assume $\mathcal{A}$ is commutative. Let $A$ be a nonempty finite subset of $\mathcal{A}$ and $B$ be a nonempty, finite subset of $U(\mathcal{A})$. Assume that $\operatorname{dim}_{\mathrm{k}}(A B) \leq \alpha \operatorname{dim}_{\mathrm{k}}(A)$ where $\alpha$ is a positive real. Then there exists a subset $X \subseteq \mathrm{k}\langle A\rangle$ such that, for any positive integer $n$,

$$
\operatorname{dim}_{\mathrm{k}}\left(X B^{n}\right) \leq \alpha^{n} \operatorname{dim}_{\mathrm{k}}(X) .
$$

In particular, if $A \subseteq U(\mathcal{A}), \operatorname{dim}_{\mathrm{k}}\left(A^{2}\right) \leq \alpha \operatorname{dim}_{\mathrm{k}}(A)$ implies that $\operatorname{dim}\left(A^{n}\right) \leq \alpha^{n} \operatorname{dim}(A)$.

Theorem 4.3 Let $A, B$ and $C$ be finite nonempty subsets of $\mathcal{A}$ such that $B \subseteq U(\mathcal{A})$. Then

$$
\operatorname{dim}_{\mathrm{k}}(A B C)^{2} \leq \operatorname{dim}_{\mathrm{k}}(A B) \operatorname{dim}_{\mathrm{k}}(B C) \max _{b \in B}\left\{\operatorname{dim}_{\mathrm{k}}(A b C)\right\} .
$$

In particular, when $\mathcal{A}$ is commutative, we have

$$
\operatorname{dim}_{\mathrm{k}}(A B C)^{2} \leq \operatorname{dim}_{\mathrm{k}}(A B) \operatorname{dim}_{\mathrm{k}}(B C) \operatorname{dim}_{\mathrm{k}}(A C) .
$$

\section{Remarks:}

1. When $\mathcal{A}$ is not commutative, the conclusion of Theorem 4.2 remains valid if we assume that $a b=b a$ for any $a \in A$ and $b \in B$.

2. The proof of the Kneser type theorems we have obtained in Section 5 (and also that of the linear Olson theorem) cannot be so easily adapted to the case of associative algebras. Indeed, given subsets $A$ and $B$ of $U(\mathcal{A}), \mathrm{k}\langle A\rangle \cap \mathrm{k}\langle B\rangle$ may have an empty intersection with $U(\mathcal{A})$. In particular, arguments based on the use of linear versions of the Dyson or Kemperman transform fail.

3. With the notation of $\S 2.3$, consider $G$ a group and $X, Y, Z$ finite subsets in $G$. Observe that $A_{X}, A_{Y}$ and $A_{Z}$ are subsets of $U(\mathbb{C}[G])$. By Theorem 4.3 , we recover Ruzsa's inequality

$$
|A B C|^{2} \leq|X Y||Y Z| \max _{y \in Y}\{|X y Z|\} .
$$

4. Theorem 4.2 also yields Theorem 1.1 also by considering subsets of $\mathbb{C}[G]$.

\section{$5 \quad$ Kneser type theorems for division rings}

In this section, $K$ is a division ring and $\mathrm{k}$ a field contained in the center of $K$.

\subsection{Assuming $A$ is commutative}

Consider a finite nonempty subset $A$ of $K^{*}$. We say that $A$ is commutative when $a a^{\prime}=a^{\prime} a$ for any $a, a^{\prime} \in A$. This then implies that the elements of $\mathrm{k}\langle A\rangle$ are pairwise commutative. Moreover the division ring $\mathbb{D}(A)$ generated by $A$ is a field. Typical examples of commutative sets are geometric progressions $A=\left\{a^{r}, a^{r+1}, \ldots, a^{r+s}\right\}$ with $r$ and $s$ integers. The following theorem is the linearization of a theorem by Diderrich [2] extending Kneser's theorem for arbitrary groups when only the subset $A$ is assumed commutative. It was shown by Hamidoune in [8] that Diderrich's result can also be derived from the original Kneser theorem in abelian group. Here we will prove our theorem without using Theorem 2.4. Also we will assume that $\mathrm{k}$ is infinite. When $K$ is finite-dimensional over $\mathrm{k}$ and $\mathrm{k}$ is finite, $K$ is a field, so we can apply Theorem 2.4 . 
Theorem 5.1 Assume $\mathrm{k}$ is infinite and every algebraic element of $K$ is separable over $\mathrm{k}$.

1. Let $A$ and $B$ be two finite nonempty subsets of $K^{*}$ such that $A$ is commutative. Then either $\operatorname{dim}_{\mathrm{k}}(A B) \geq \operatorname{dim}_{\mathrm{k}}(A)+\operatorname{dim}_{\mathrm{k}}(B)-1$ or $\mathrm{k}\langle A B\rangle$ is left periodic.

2. Let $A_{1}, \ldots, A_{n}$ be a collection of finite nonempty subsets of $K^{*}$ such that $A_{1}, \ldots, A_{n-1}$ are commutative. Then either $\operatorname{dim}_{\mathrm{k}}\left(A_{1} \cdots A_{n}\right) \geq \sum_{i=1}^{n} \operatorname{dim}_{\mathrm{k}}\left(A_{i}\right)-(n-1)$ or $\mathrm{k}\left\langle A_{1} \cdots A_{n}\right\rangle$ is periodic.

Remark: In the linear Olson theorem established in [3] (where $A$ is not assumed commutative), we only obtain that $\operatorname{dim}_{\mathrm{k}}(A B) \geq \operatorname{dim}_{\mathrm{k}}(A)+\operatorname{dim}_{\mathrm{k}}(B)-1$, or $\mathrm{k}\langle A B\rangle$ contains a (left or right) periodic subspace.

To prove the theorem, we need to adapt the arguments of [4] to our noncommutative situation. We begin with the following lemma based on the linear Dyson transform.

Lemma 5.2 Let $A$ and $B$ be two finite, nonempty subsets of $K^{*}$ such that $A$ is commutative. Then, for each nonzero $a \in \mathrm{k}\langle A\rangle$, there exists a (commutative) subfield $H_{a}$ of $K$ such that $\mathrm{k} \subseteq H_{a} \subseteq \mathbb{D}(A)$ and a vector space $V_{a} \neq\{0\}$ contained in $\mathrm{k}\langle A B\rangle$ such that $H_{a} V_{a}=V_{a}, \mathrm{k}\langle a B\rangle \subseteq V_{a}$ and

$$
\operatorname{dim}_{\mathrm{k}}\left(V_{a}\right)+\operatorname{dim}_{\mathrm{k}}\left(H_{a}\right) \geq \operatorname{dim}_{\mathrm{k}}(A)+\operatorname{dim}_{\mathrm{k}}(B) .
$$

Proof. By replacing $A$ by $A^{\prime}=a^{-1} A$, we can assume $a=1$. Indeed, if there exist a subfield $H \subseteq \mathbb{D}\left(A^{\prime}\right)$ and a vector space $V \neq\{0\}$ contained in $\mathrm{k}\left\langle A^{\prime} B\right\rangle$ such that $H V=V$ and $\mathrm{k}\langle B\rangle \subseteq V$ with

$$
\operatorname{dim}_{\mathrm{k}}(V)+\operatorname{dim}_{\mathrm{k}}(H) \geq \operatorname{dim}_{\mathrm{k}}\left(A^{\prime}\right)+\operatorname{dim}_{\mathrm{k}}(B),
$$

it suffices to take $V_{a}=a V$ and $H_{a}=H \subseteq \mathbb{D}\left(A^{\prime}\right) \subseteq \mathbb{D}(A)$. Since $H \subseteq \mathbb{D}(A)$, we must have $H a=a H$ for any $a \in A$ and $H\left(V_{a}\right)=H(a V)=a H V=a V=V_{a}$. Moreover $\mathrm{k}\langle a B\rangle=a \mathrm{k}\langle B\rangle \subseteq a V=V_{a}$ and $\operatorname{dim}_{\mathrm{k}}\left(V_{a}\right)+\operatorname{dim}_{\mathrm{k}}\left(H_{a}\right) \geq \operatorname{dim}_{\mathrm{k}}(A)+\operatorname{dim}_{\mathrm{k}}(B)$ because $\operatorname{dim}_{\mathrm{k}}\left(V_{a}\right)=\operatorname{dim}_{\mathrm{k}}(V)$ and $H_{a}=H$.

We can also assume that $1 \in B$ by replacing $B$ by $B^{\prime}=B b^{-1}$. Indeed, if there exist a subfield $H^{\prime} \subseteq \mathbb{D}(A)$ and a vector space $V^{\prime} \neq\{0\}$ contained in $\mathrm{k}\left\langle A B^{\prime}\right\rangle$ such that $H^{\prime} V^{\prime}=V^{\prime}$ and $\mathrm{k}\left\langle B^{\prime}\right\rangle \subseteq V^{\prime}$ with

$$
\operatorname{dim}_{\mathrm{k}}\left(V^{\prime}\right)+\operatorname{dim}_{\mathrm{k}}\left(H^{\prime}\right) \geq \operatorname{dim}_{\mathrm{k}}(A)+\operatorname{dim}_{\mathrm{k}}\left(B^{\prime}\right),
$$

it suffices to take $V=V^{\prime} b$ and $H=H^{\prime}$. We will have then $V=V^{\prime} b \subseteq \mathrm{k}\left\langle A B^{\prime}\right\rangle b=\mathrm{k}\langle A B\rangle$, $H V=H\left(V^{\prime} b\right)=\left(H V^{\prime}\right) b=V^{\prime} b=V, \mathrm{k}\langle B\rangle=\mathrm{k}\left\langle B^{\prime}\right\rangle b \subseteq V^{\prime} b=V$ and

$$
\operatorname{dim}_{\mathrm{k}}(V)+\operatorname{dim}_{\mathrm{k}}(H) \geq \operatorname{dim}_{\mathrm{k}}(A)+\operatorname{dim}_{\mathrm{k}}(B)
$$

since $\operatorname{dim}_{\mathrm{k}}(B)=\operatorname{dim}_{\mathrm{k}}\left(B^{\prime}\right)$ and $\operatorname{dim}_{\mathrm{k}}(V)=\operatorname{dim}_{\mathrm{k}}\left(V^{\prime}\right)$.

We thus assume in the remainder of the proof that $1 \in A \cap B$ and proceed by induction on $\operatorname{dim}_{\mathrm{k}}(A)$. When $\operatorname{dim}_{\mathrm{k}}(A)=1$, we have $\mathrm{k}\langle A\rangle=\mathrm{k}=\mathbb{D}(A)$. It suffices to take $V_{1}=V=\mathrm{k}\langle B\rangle \neq\{0\}$ and $H_{a}=H=\mathrm{k}=\mathbb{D}(A)$. Assume $\operatorname{dim}_{\mathrm{k}}(A)>1$. Given $e \in \mathrm{k}\langle B\rangle$ such that $e \neq 0$, define $A(e)$ and $B(e)$ to be finite subsets of $K^{*}$ such that

$$
\mathrm{k}\langle A(e)\rangle=\mathrm{k}\langle A\rangle \cap \mathrm{k}\langle B\rangle e^{-1} \text { and } \mathrm{k}\langle B(e)\rangle=\mathrm{k}\langle B\rangle+\mathrm{k}\langle A\rangle e .
$$

Observe that $\mathrm{k}\langle A(e)\rangle$ and $\mathrm{k}\langle B(e)\rangle$ contain $\mathrm{k}$ since $1 \in A \cap B$. Thus we may and do assume that $1 \in A(e) \cap B(e)$. Moreover, $\mathrm{k}\langle A(e)\rangle \mathrm{k}\langle B(e)\rangle$ is contained in $\mathrm{k}\langle A B\rangle$. Indeed, for $v \in \mathrm{k}\langle A\rangle \cap \mathrm{k}\langle B\rangle e^{-1}$ 
and $w \in \mathrm{k}\langle B\rangle$, we have $v w \in \mathrm{k}\langle A\rangle \mathrm{k}\langle B\rangle \subseteq \mathrm{k}\langle A B\rangle$ because $v \in \mathrm{k}\langle A\rangle$. Set $v=z e^{-1}$ with $z \in \mathrm{k}\langle B\rangle$. If $w \in \mathrm{k}\langle A\rangle e$, we have $v w \in z e^{-1} \mathrm{k}\langle A\rangle e$. But $z e^{-1} \in \mathrm{k}\langle A\rangle$ and $A$ is commutative. Therefore, $v w \in \mathrm{k}\langle A\rangle z e^{-1} e=\mathrm{k}\langle A\rangle z \subseteq \mathrm{k}\langle A\rangle \mathrm{k}\langle B\rangle \subseteq \mathrm{k}\langle A B\rangle$. In particular, $\operatorname{dim}_{\mathrm{k}}(A(e) B(e)) \leq \operatorname{dim}_{\mathrm{k}}(A B)$. We get

$$
\begin{aligned}
& \operatorname{dim}_{\mathrm{k}}(A(e))+\operatorname{dim}_{\mathrm{k}}(B(e))=\operatorname{dim}_{\mathrm{k}}\left(\mathrm{k}\langle A\rangle \cap \mathrm{k}\langle B\rangle e^{-1}\right)+\operatorname{dim}_{\mathrm{k}}(\mathrm{k}\langle B\rangle+\mathrm{k}\langle A\rangle e)= \\
& \quad \operatorname{dim}_{\mathrm{k}}(\mathrm{k}\langle A\rangle e \cap \mathrm{k}\langle B\rangle)+\operatorname{dim}_{\mathrm{k}}(\mathrm{k}\langle B\rangle+\mathrm{k}\langle A\rangle e)=\operatorname{dim}_{\mathrm{k}}(A e)+\operatorname{dim}_{\mathrm{k}}(B)=\operatorname{dim}_{\mathrm{k}}(A)+\operatorname{dim}_{\mathrm{k}}(B) .
\end{aligned}
$$

Also $A(e) \subseteq \mathrm{k}\langle A\rangle$.

Assume $\mathrm{k}\langle A(e)\rangle=\mathrm{k}\langle A\rangle$ for any nonzero $e \in \mathrm{k}\langle B\rangle$. Then $\mathrm{k}\langle A\rangle e \subseteq \mathrm{k}\langle B\rangle$ for any nonzero $e \in \mathrm{k}\langle B\rangle$. Thus $\mathrm{k}\langle A B\rangle \subseteq \mathrm{k}\langle B\rangle$. Since $1 \in A$, we have in fact $\mathrm{k}\langle A B\rangle=\mathrm{k}\langle B\rangle$. The sub division $\operatorname{ring} H=\mathbb{D}(A)$ is a field since $A$ is commutative and it contains $\mathrm{k}$ since $1 \in A$. Take $V=\mathrm{k}\langle B\rangle \neq\{0\}$. Then $H V=V$ since $A V=V$. We clearly have $V=\mathrm{k}\langle A B\rangle$ and $B \subseteq V$ as desired.

Now assume $\mathrm{k}\langle A(e)\rangle \neq \mathrm{k}\langle A\rangle$ for at least one nonzero $e \in \mathrm{k}\langle A\rangle$. Then $0<\operatorname{dim}_{\mathrm{k}}(A(e))<\operatorname{dim}_{\mathrm{k}}(A)$ and $1 \in A(e) \cap B(e)$. By our induction hypothesis, there exist a subfield $H$ of $\mathbb{D}(A(e)) \subseteq \mathbb{D}(A)$ containing $\mathrm{k}$ and a nonzero k-vector space $V \subseteq \mathrm{k}\langle A(e) B(e)\rangle \subseteq \mathrm{k}\langle A B\rangle$, such that $H V=V$ and $\mathrm{k}\langle B\rangle \subseteq \mathrm{k}\langle B(e)\rangle \subseteq V$ with

$$
\operatorname{dim}_{\mathrm{k}}(V)+\operatorname{dim}_{\mathrm{k}}(H) \geq \operatorname{dim}_{\mathrm{k}}(A(e))+\operatorname{dim}_{\mathrm{k}}(B(e))=\operatorname{dim}_{\mathrm{k}}(A)+\operatorname{dim}_{\mathrm{k}}(B) .
$$

The subfield $H \subseteq \mathbb{D}(A)$ and the nonzero space $V \supset \mathrm{k}\langle B\rangle$ satisfy the statement of the lemma for the pair of subsets $A$ and $B$ which concludes the proof.

As in the proof of Theorem 2.4, we also need the following lemma, which is an application of the Vandermonde determinant.

Lemma 5.3 Let $V$ be a $n$-dimensional vector space over the infinite field $\mathrm{k}$. Assume $x_{1}, \ldots, x_{n}$ form a basis of $V$ over $\mathrm{k}$. Then any $n$ vectors in the set

$$
\left\{x_{1}+\alpha x_{2}+\cdots+\alpha^{n-1} x_{n} \mid \alpha \in \mathrm{k}\right\}
$$

form a basis of $V$ over $\mathrm{k}$.

The proof of Theorem 5.1 requires a last lemma. Consider a field extension $F$ over the infinite field $\mathrm{k}$. Let $y_{1}, \ldots, y_{r}$ be algebraically independent indeterminates. Set $\mathrm{k}^{\prime}=\mathrm{k}\left(y_{1}, \ldots, y_{r}\right)$ and $F^{\prime}=F\left(y_{1}, \ldots, y_{r}\right)$.

Lemma 5.4 Assume $\operatorname{dim}_{\mathrm{k}^{\prime}}\left(F^{\prime}\right)$ is finite. Then $\operatorname{dim}_{\mathrm{k}}(F)=\operatorname{dim}_{\mathrm{k}^{\prime}}\left(F^{\prime}\right)$.

Proof. Let $b_{1}, \ldots, b_{n}$ be elements in $F$ linearly independent over k. Assume there exists nonzero elements $P_{1}, \ldots, P_{n}$ in $\mathrm{k}^{\prime}$ such that

$$
\sum_{i=1}^{n} P_{i} b_{i}=0 .
$$

By multiplying by an overall polynomial of $\mathrm{k}^{\prime}$, we can assume that the $P_{i}$ 's belong to $k\left[y_{1}, \ldots, y_{r}\right]$. Set $I=\left\{i \in\{1, \ldots n\} \mid P_{i} \neq 0\right\}$. Assume $I$ is nonempty. Set $P=\prod_{i \in I} P_{i}$ and consider $V=$ $\left\{\left(u_{1}, \ldots, u_{r}\right) \in \mathrm{k}^{r} \mid P\left(u_{1}, \ldots, u_{r}\right)=0\right\}$. Since $P \neq 0$ and $\mathrm{k}$ is infinite, $V \neq k^{n}$. Therefore, there 
exists $\left(u_{1}, \ldots, u_{r}\right)$ in $\mathrm{k}^{r}$ such that $P\left(u_{1}, \ldots, u_{r}\right) \neq 0$. Then for any $i \in I$, we have $P_{i}\left(u_{1}, \ldots, u_{r}\right) \neq 0$ and

$$
\sum_{i=1}^{n} P_{i}\left(u_{1}, \ldots, u_{r}\right) b_{i}=0
$$

which contradicts our assumption that $b_{1}, \ldots, b_{n}$ are linearly independent over $\mathrm{k}$. We have proved that $\operatorname{dim}_{\mathrm{k}}(F) \leq \operatorname{dim}_{\mathrm{k}^{\prime}}\left(F^{\prime}\right)$. In particular, $\operatorname{dim}_{\mathrm{k}}(F)$ is finite. We assume from now on that $\operatorname{dim}_{\mathrm{k}}(F)=$ $n$ and $\left\{b_{1}, \ldots, b_{n}\right\}$ is a basis of $F$ over k. Let

$$
E=\bigoplus_{i=1}^{n} \mathrm{k}^{\prime} b_{i}
$$

Clearly $E$ is a $n$-dimensional $\mathrm{k}^{\prime}$-space closed under multiplication. So by Lemma 2.2 , $E$ is a subfield of $F^{\prime}$. Moreover $E$ contains the polynomial ring $F\left[y_{1}, \ldots, y_{r}\right]$ since each element of $F$ decomposes as a k-linear combination of the $b_{i}$ 's. This implies that $E=F^{\prime}$ and $\operatorname{dim}_{\mathrm{k}}(F) \geq \operatorname{dim}_{\mathrm{k}^{\prime}}\left(F^{\prime}\right)$. So $\operatorname{dim}_{\mathrm{k}}(F)=\operatorname{dim}_{\mathrm{k}^{\prime}}\left(F^{\prime}\right)$ as desired.

We are now ready to prove 5.1 .

Proof. (of Theorem 5.1)

1: Let $\mathcal{B}=\left\{x_{1}, \ldots, x_{n}\right\}$ be a basis of $\mathrm{k}\langle A\rangle$. For any $\alpha \in \mathrm{k}$, set $x_{\alpha}=x_{1}+\alpha x_{2}+\cdots+\alpha^{n-1} x_{n}$. Observe that $x_{\alpha} \neq 0$. Since $\mathrm{k}$ is infinite and by Lemma 5.2, there exist a subfield $H_{\alpha}$ such that $\mathrm{k} \subseteq H_{\alpha} \subseteq \mathbb{D}(A) \subseteq K$ and a k-vector space $V_{\alpha} \subseteq \mathrm{k}\langle A B\rangle$ with $x_{\alpha} B \subseteq V_{\alpha}, H_{\alpha} V_{\alpha}=V_{\alpha}$ and $\operatorname{dim}_{\mathrm{k}}\left(V_{\alpha}\right)+\operatorname{dim}_{\mathrm{k}}\left(H_{\alpha}\right) \geq \operatorname{dim}_{\mathrm{k}}(A)+\operatorname{dim}_{\mathrm{k}}(B)$. Since $V_{\alpha} \neq\{0\}$ and $H_{\alpha}$ stabilizes $V_{\alpha} \subseteq \mathrm{k}\langle A B\rangle$, there exists a nonzero vector $v \in V_{\alpha}$ such that $H_{\alpha} v \subseteq \mathrm{k}\langle A B\rangle$. Hence $H_{\alpha} \subseteq v^{-1} \mathrm{k}\langle A B\rangle$ and $\operatorname{dim}_{\mathrm{k}}\left(H_{\alpha}\right)$ is finite. Therefore $H_{\alpha} \subseteq \mathbb{D}(A)$ is a finite field extension of k. Let $F$ be the algebraic closure of $\mathrm{k}$ in $\mathbb{D}(A)$. The elements of $H_{\alpha}$ belong to $\mathbb{D}(A)$ and are algebraic over $\mathrm{k}$ since $\operatorname{dim}_{\mathrm{k}}\left(H_{\alpha}\right)$ is finite. Therefore $H_{\alpha} \subseteq F$ for any $\alpha \in \mathrm{k}$.

The field $\mathbb{D}(A)$ is finitely generated by $x_{1}, \ldots, x_{n}$. Therefore, if $F=\mathbb{D}(A)$, each $x_{i}$ is algebraic over $\mathrm{k}$ and $\operatorname{dim}_{\mathrm{k}}(F)$ is finite. If $F \varsubsetneqq \mathbb{D}(A)$, we can choose a family $y_{1}, \ldots, y_{r}$ in $\mathbb{D}(A)$ such that $\mathrm{k}^{\prime}=\mathrm{k}\left(y_{1}, \ldots, y_{r}\right)$ is purely transcendental over $\mathrm{k}$ and $\mathbb{D}(A)$ is algebraic finitely generated over $\mathrm{k}^{\prime}$. Then $\operatorname{dim}_{\mathrm{k}^{\prime}}(\mathbb{D}(A))$ is finite. Thus by Lemma $5.4 \operatorname{dim}_{\mathrm{k}}(F)=\operatorname{dim}_{\mathrm{k}^{\prime}}\left(F\left(y_{1}, \ldots, y_{r}\right)\right) \leq \operatorname{dim}_{\mathrm{k}^{\prime}}(\mathbb{D}(A))$ is finite. So in both cases, we obtain that $\operatorname{dim}_{\mathrm{k}}(F)$ is finite.

By the separability hypothesis, we obtain that the extension $F$ is separable over k. Thus, it only admits a finite number of intermediate extensions. There should exist $n$ distinct elements $\alpha_{1}, \ldots, \alpha_{n}$ in $\mathrm{k}$ such that

$$
H_{\alpha_{1}}=H_{\alpha_{2}}=\cdots=H_{\alpha_{n}}=H .
$$

By Lemma 5.3, $x_{\alpha_{1}}, \ldots, x_{\alpha_{n}}$ form a basis of $\mathrm{k}\langle A\rangle$ over $\mathrm{k}$. We thus have $\mathrm{k}\langle A B\rangle=\sum_{i=1}^{n} x_{\alpha_{i}} \mathrm{k}\langle B\rangle \subseteq$ $\sum_{i=1}^{n} V_{\alpha_{i}}$ since $x_{\alpha_{i}} \mathrm{k}\langle B\rangle \subseteq V_{\alpha_{i}}$ for any $i=1, \ldots, n$. On the other hand, $V_{\alpha_{i}} \subseteq \mathrm{k}\langle A B\rangle$ for any $i=1, \ldots, n$. Hence $\mathrm{k}\langle A B\rangle=\sum_{i=1}^{n} V_{\alpha_{i}}$ is stabilized by $H$. If $\mathrm{k} \varsubsetneqq H$, then $\mathrm{k}\langle A B\rangle$ is periodic and we are done. Otherwise, $\mathrm{k}=H$ and we have

$$
\operatorname{dim}_{\mathrm{k}}(A)+\operatorname{dim}_{\mathrm{k}}(B) \leq \operatorname{dim}_{\mathrm{k}}\left(V_{\alpha_{i}}\right)+1
$$

for any $i=1, \ldots, n$. Since $V_{\alpha_{i}} \subseteq \mathrm{k}\langle A B\rangle$, we obtain

$$
\operatorname{dim}_{\mathrm{k}}(A)+\operatorname{dim}_{\mathrm{k}}(B) \leq \operatorname{dim}_{\mathrm{k}}(A B)+1
$$

as desired. 
2: In part (II) of the proof of Theorem 2.7, we do not use any commutativity hypothesis on $K$. So both assertions of Theorem 5.1 are equivalent by exactly the same arguments.

\section{Remarks:}

1. When $B$ is assumed commutative, we have a similar statement by replacing left periodicity by right periodicity.

2. Also observe that the separability hypothesis is always satisfied when $\mathrm{k}$ has characteristic 0 .

3. Theorem 5.1 means that when $A$ is commutative and $\operatorname{dim}_{\mathrm{k}}(A B) \leq \operatorname{dim}_{\mathrm{k}}(A)+\operatorname{dim}_{\mathrm{k}}(B)-2$, $\mathrm{k}\langle A B\rangle$ is an left $H$-module. When $A=B$ or $A^{-1}=B$, this suggests that spaces $\mathrm{k}\langle A\rangle$ with $\operatorname{dim}_{\mathrm{k}}\left(A^{2}\right)=O(\operatorname{dim} A)$ should have interesting properties related to some $H$-modules of $K$ where $H$ is a subdivision ring of $K$. We will make this observation precise in the following paragraphs.

Theorem 5.1 also permits one to construct $\mathrm{k}$-subspaces in $K$ containing subdivision rings. Assume $\operatorname{dim}_{\mathrm{k}}(K)$ is finite and let $a_{1}, \ldots, a_{n}$ be a sequence of elements in $K^{*}$ distinct from 1 (with repetition allowed). For any nonempty subset $S \subseteq\{1, \ldots, n\}$, write $a_{S}:=\prod_{i \in S} a_{i}$. Denote by $V$ the k-subspace in $K$ generated by the elements $a_{S}$ when $S$ runs over the nonempty subsets of $\{1, \ldots, n\}$.

Corollary 5.5 Assume $\mathrm{k}$ is infinite, $\operatorname{dim}_{\mathrm{k}}(K)=n>1$ and every element of $K$ is separable over $\mathrm{k}$. Then, with the above notation, the space $V$ contains a sub division ring $H \supsetneqq \mathrm{k}$.

Proof. For any $i=1, \ldots, n$, set $A_{i}=\left\{1, a_{i}\right\}$ and $V^{\prime}=\mathrm{k}\left\langle A_{1} \cdots A_{n}\right\rangle$. Write $p_{i}=a_{1} \cdots a_{i}$ for any $i=1, \ldots, n$. If $p_{1}, \ldots, p_{n}$ are linearly independent, $V=K$ since $\operatorname{dim}_{\mathrm{k}}(K)=n$. In particular, $1 \in V$.

Now assume $p_{1}, \ldots, p_{n}$ are not linearly independent. There exist $i_{0} \in\{1, \ldots, n\}$ and elements $\alpha_{i}, i=i_{0}, \ldots, n$, in $\mathrm{k}$ such that

$$
\sum_{i=i_{0}}^{n} \alpha_{i} p_{i}=0 \text { and } \alpha_{i_{0}} \neq 0
$$

Dividing by $\alpha_{i_{0}} p_{i_{0}}$, we obtain

$$
1=-\sum_{i=i_{0}+1}^{n} \frac{\alpha_{i}}{\alpha_{i_{0}}} a_{i_{0}+1} \cdots a_{i}
$$

We thus also obtain that $1 \in V$.

We have proved that $1 \in V$. This implies that $V^{\prime}=V$. If $V$ is left periodic, there exists a subdivision ring $H \subseteq K$ such that $H \supseteqq \mathrm{k}$ and $H V \subseteq V$. Since $1 \in V$, we have $H \subseteq V$ as desired. We can thus assume that $V=\mathrm{k}\left\langle A_{1} \cdots A_{n}\right\rangle$ is not periodic. As the sets $A_{i}, i=1, \ldots n$ are commutative, we can apply 2 of Theorem 2.7 which gives

$$
\operatorname{dim}_{\mathrm{k}}\left(A_{1} \cdots A_{n}\right) \geq \sum_{i=1}^{n} \operatorname{dim}_{\mathrm{k}}\left(A_{i}\right)-(n-1) \geq 2 n-(n-1) \geq n+1 .
$$

We thus obtain a contradiction with the hypothesis $\operatorname{dim}_{\mathrm{k}}(K)=n$. 


\subsection{Linear hamidoune's connectivity}

The notion of connectivity for a subset $S$ of a group $G$ was developed by Hamidoune in [7]. As suggested by Tao in [18, it is interesting to generalize Hamidoune's definition by introducing an additional parameter $\lambda$. The purpose of this paragraph is to define a natural linear version of this connectivity used in [18] suited for the k-subspaces $V$ in $K$, where $K$ is a division ring containing $\mathrm{k}$ in its center. Assume $V$ is a finite-dimensional fixed k-subspace of $K$ and $\lambda$ is a real parameter. For any finite-dimensional k-subspace $W$ of $K$, we define

$$
c(W):=\operatorname{dim}_{\mathrm{k}}(W V)-\lambda \operatorname{dim}_{\mathrm{k}}(W) .
$$

For any $x \in K^{*}$, we have immediately that $c(x W)=c(W)$.

Lemma 5.6 For any finite-dimensional subspaces $W_{1}, W_{2}$ and $V$ of $K$, we have

$$
c\left(W_{1}+W_{2}\right)+c\left(W_{1} \cap W_{2}\right) \leq c\left(W_{1}\right)+c\left(W_{2}\right) .
$$

Proof. We have

$$
\operatorname{dim}_{\mathrm{k}}\left(W_{1}+W_{2}\right)+\operatorname{dim}_{\mathrm{k}}\left(W_{1} \cap W_{2}\right)=\operatorname{dim}_{\mathrm{k}}\left(W_{1}\right)+\operatorname{dim}_{\mathrm{k}}\left(W_{2}\right)
$$

and

$$
\operatorname{dim}_{\mathrm{k}}\left(\mathrm{k}\left\langle W_{1} V\right\rangle+\mathrm{k}\left\langle W_{2} V\right\rangle\right)+\operatorname{dim}_{\mathrm{k}}\left(\mathrm{k}\left\langle W_{1} V\right\rangle \cap \mathrm{k}\left\langle W_{2} V\right\rangle\right)=\operatorname{dim}_{\mathrm{k}}\left(W_{1} V\right)+\operatorname{dim}_{\mathrm{k}}\left(W_{2} V\right) .
$$

Observe that $\mathrm{k}\left\langle\left(W_{1}+W_{2}\right) \cdot V\right\rangle=\mathrm{k}\left\langle W_{1} V\right\rangle+\mathrm{k}\left\langle W_{2} V\right\rangle$ and $\mathrm{k}\left\langle\left(W_{1} \cap W_{2}\right) \cdot V\right\rangle \subseteq \mathrm{k}\left\langle W_{1} V\right\rangle \cap \mathrm{k}\left\langle W_{2} V\right\rangle$. This gives

$$
\left.\operatorname{dim}_{\mathrm{k}}\left(\left(W_{1}+W_{2}\right) \cdot V\right)+\operatorname{dim}_{\mathrm{k}}\left(W_{1} \cap W_{2}\right) \cdot V\right) \leq \operatorname{dim}_{\mathrm{k}}\left(W_{1} V\right)+\operatorname{dim}_{\mathrm{k}}\left(W_{2} V\right) .
$$

We then obtain the desired equality by subtracting from (12), $\lambda$ copies of (11).

Similarly to [7], we define the connectivity $\kappa=\kappa(V)$ as the infimum of $c(W)$ over all finitedimensional, nonzero k-subspaces of $K$. A fragment of $V$ is a finite-dimensional k-subspace of $K$ which attains the infimum $\kappa$. An atom of $V$ is a fragment of minimal dimension. Since $c(x W)=$ $c(W)$, any left translate of a fragment is a fragment and any left translate of an atom is an atom. Since $\operatorname{dim}_{\mathrm{k}}(W V) \geq \operatorname{dim}_{\mathrm{k}}(W)$, we have

$$
c(W) \geq(1-\lambda) \operatorname{dim}_{\mathrm{k}}(W) .
$$

We observe that when $\lambda<1, c(W)$ is always positive and takes a discrete set of values. Therefore, when $\lambda<1$, there exists at least one fragment and at least one atom. In the remainder of this paragraph we will assume that $\lambda<1$. Let $W_{1}$ and $W_{2}$ be two fragments with nonzero intersection. By the previous lemma, we derive

$$
c\left(W_{1}+W_{2}\right)+c\left(W_{1} \cap W_{2}\right) \leq c\left(W_{1}\right)+c\left(W_{2}\right) \leq 2 \kappa .
$$

Since $W_{1}+W_{2}$ and $W_{1} \cap W_{2}$ are finite-dimensional and not reduced to $\{0\}$, we must have $c\left(W_{1}+\right.$ $\left.W_{2}\right) \geq \kappa$ and $c\left(W_{1} \cap W_{2}\right) \geq \kappa$. Hence $c\left(W_{1}+W_{2}\right)=c\left(W_{1} \cap W_{2}\right)=\kappa$. This means that $W_{1}+W_{2}$ and $W_{1} \cap W_{2}$ are also fragments. If we assume now that $W_{1}$ and $W_{2}$ are atoms, we obtain that $W_{1}=W_{2}$ or $W_{1} \cap W_{2}=\{0\}$. 
Proposition 5.7 Let $K$ be a division ring containing $\mathrm{k}$ in its center and let $V$ be a finite-dimensional k-subspace .

1. There exists a unique atom $H$ containing 1 for $V$.

2. This atom is a division ring containing $\mathrm{k}$ in its center.

3. Moreover the atoms of $V$ are the right modules $x H$ where $x$ runs over $K^{*}$.

Proof. Since there exists at least one atom and the left translate of any atom is an atom, there exists one atom $H$ containing 1 . Now, this atom must be unique because atoms are equal or with an intersection reduced to $\{0\}$. In particular, for any $x \in K, H=x H$ or $H \cap x H=\{0\}$. We claim that this implies that $H$ is a division ring. Indeed, for any $h \in H, H \cap h^{-1} H$ contains 1 for $1 \in H$. Therefore $h^{-1} H=H$ and $H=h H$. So $H$ is stable under multiplication. We then deduce that $H$ is a division ring as in the proof of Lemma 2.1. Finally, given any atom $W$ of $V$, we must have $w^{-1} W=H$ for any nonzero $w \in W$ since $H$ is the unique atom containing 1 and $w^{-1} H$ is an atom.

\subsection{Tao's theorem for division rings}

We say that $V=\mathrm{k}\langle A\rangle$, where $A$ is a finite subset of $K^{*}$, is a space of small doubling, when $\operatorname{dim}_{\mathrm{k}}\left(A^{2}\right)=O\left(\operatorname{dim}_{\mathrm{k}}(A)\right)$. Simplest examples of spaces of small doubling are the spaces $V=\mathrm{k}\langle A\rangle$ containing 1 and such that $\operatorname{dim}_{\mathrm{k}}\left(A^{2}\right)=\operatorname{dim}_{\mathrm{k}}(A)$. Then by Lemma 2.1, $V$ is a division ring containing $\mathrm{k}$. In general, a space of small doubling $\mathrm{k}\langle A\rangle$ is not a division ring and neither a left nor right $H$-module for a division ring $\mathrm{k} \subseteq H \subseteq K$. The following theorem, which is the linear version of Theorem 1.2 in [18], permits to study the spaces of small doubling in $K$.

Theorem 5.8 Let $K$ be a division ring containing the field $\mathrm{k}$ in its center. Consider finitedimensional $\mathrm{k}$-subspaces $V$ and $W$ of $K$ such that $\operatorname{dim}_{\mathrm{k}}(W) \geq \operatorname{dim}_{\mathrm{k}}(V)$ and $\operatorname{dim}_{\mathrm{k}}(W V) \leq(2-$ $\varepsilon) \operatorname{dim}_{\mathrm{k}}(V)$ for some real $\varepsilon$ such that $0<\varepsilon<2$. Then one of the following statements holds:

- There exists a division ring $H$ containing $\mathrm{k}$ such that $\operatorname{dim}_{\mathrm{k}}(H) \leq\left(\frac{2}{\varepsilon}-1\right) \operatorname{dim}_{\mathrm{k}}(V)$, and $V$ is contained in a left module $H x$ with $x \in K^{*}$.

- There exists a division ring $H$ containing $\mathrm{k}$ such that $\operatorname{dim}_{\mathrm{k}}(H) \leq\left(\frac{2}{\varepsilon}-1\right) /\left(\frac{2}{\varepsilon}+1\right) \operatorname{dim}_{\mathrm{k}}(V)$, and a finite subset $X$ of $K^{*}$ with $|X| \leq \frac{2}{\varepsilon}-1$ such that $V \subseteq \bigoplus_{x \in X} H x$.

Proof. We apply linear Hamidoune connectivity with $\lambda=1-\frac{\varepsilon}{2}$. We have by (13) $c(U) \geq$ $\frac{\varepsilon}{2} \operatorname{dim}_{\mathrm{k}}(U)$ for any k-subspace $U$. This can be rewritten as

$$
\operatorname{dim}_{\mathrm{k}}(U) \leq \frac{2}{\varepsilon} c(U)
$$

We also get

$$
c(W):=\operatorname{dim}_{\mathrm{k}}(W V)-\left(1-\frac{\varepsilon}{2}\right) \operatorname{dim}_{\mathrm{k}}(W) \leq(2-\varepsilon) \operatorname{dim}_{\mathrm{k}}(V)-\left(1-\frac{\varepsilon}{2}\right) \operatorname{dim}_{\mathrm{k}}(V)=\left(1-\frac{\varepsilon}{2}\right) \operatorname{dim}_{\mathrm{k}}(V) .
$$

since $\operatorname{dim}_{\mathrm{k}}(W V) \leq(2-\varepsilon) \operatorname{dim}_{\mathrm{k}}(V)$ and $\operatorname{dim}_{\mathrm{k}}(W) \geq \operatorname{dim}_{\mathrm{k}}(V)$. By Proposition 5.7, the unique atom containing 1 is a division ring $H$. By definition of an atom, we should have

$$
\kappa=c(H) \leq c(W) \leq\left(1-\frac{\varepsilon}{2}\right) \operatorname{dim}_{\mathrm{k}}(V) .
$$


We therefore obtain, by using (14) with $U=H$, that

$$
\operatorname{dim}_{\mathrm{k}}(H) \leq \frac{2}{\varepsilon} c(H) \leq \frac{2}{\varepsilon} c(W) \leq\left(\frac{2}{\varepsilon}-1\right) \operatorname{dim}_{\mathrm{k}}(V) .
$$

If $V$ is contained in a left module $H x$, we are done. Assume $V$ intersects at least two such left $H$-modules. By using that $c(H)=\operatorname{dim}_{\mathrm{k}}(H V)-\left(1-\frac{\varepsilon}{2}\right) \operatorname{dim}_{\mathrm{k}}(H)$ and the previous inequality $c(H) \leq\left(1-\frac{\varepsilon}{2}\right) \operatorname{dim}_{\mathrm{k}}(V)$, we get

$$
\operatorname{dim}_{\mathrm{k}}(H V) \leq\left(1-\frac{\varepsilon}{2}\right) \operatorname{dim}_{\mathrm{k}}(V)+\left(1-\frac{\varepsilon}{2}\right) \operatorname{dim}_{\mathrm{k}}(H) .
$$

Since $V$ intersects at least two left $H$-modules, we must have $\operatorname{dim}_{\mathrm{k}}(H V) \geq 2 \operatorname{dim}_{\mathrm{k}}(H)$. By using (15), this gives

$$
\operatorname{dim}_{\mathrm{k}}(H) \leq \frac{1-\frac{\varepsilon}{2}}{1+\frac{\varepsilon}{2}} \operatorname{dim}_{\mathrm{k}}(V)<\operatorname{dim}_{\mathrm{k}}(V) .
$$

We can also bound $\operatorname{dim}_{\mathrm{k}}(V)$ by $\operatorname{dim}_{\mathrm{k}}(H V)$ in (15). This yields

$$
\operatorname{dim}_{\mathrm{k}}(H V) \leq\left(\frac{2}{\varepsilon}-1\right) \operatorname{dim}_{\mathrm{k}}(H) .
$$

Now $\mathrm{k}\langle H V\rangle$ is left $H$-invariant and finite-dimensional because $\operatorname{dim}_{\mathrm{k}}(H V) \leq \operatorname{dim}_{\mathrm{k}}(H) \operatorname{dim}_{\mathrm{k}}(V) \leq$ $\operatorname{dim}_{\mathrm{k}}(V)^{2}$. By Lemma 2.2, there thus exists a finite subset $X$ of $K^{*}$ such that

$$
\mathrm{k}\langle H V\rangle=\bigoplus_{x \in X} H x
$$

By (16), we should have $|X| \leq\left(\frac{2}{\varepsilon}-1\right)$. Moreover $V \subseteq \mathrm{k}\langle H V\rangle$, which concludes the proof.

Remark: When $\operatorname{dim}_{\mathrm{k}}\left(A^{2}\right) \leq(2-\varepsilon) \operatorname{dim}_{\mathrm{k}}(A)$, we can apply Theorem 5.8 with $V=W=\mathrm{k}\langle A\rangle$ and obtain information on the covering of $V$ by left $H$-modules. This can be interpreted as a description of subspaces with small doubling similar to the description of sets with small doubling obtained in [18.

Acknowledgments: The author thanks both anonymous referees for their very careful reading of the paper and their many valuable corrections and comments.

\section{References}

[1] S. A. Amitsur, Finite subgroups of division rings, Trans. Ams. 80 (1955), 361-386 .

[2] G. T. Diderrich, On Kneser's addition theorem in groups, Proc. Ams. 38 (1973), 443-451.

[3] S. Eliahou. and C. Lecouvey, On linear versions of some addition theorems, Linear Algebra and multilinear algebra, 57 (2009), 759-775.

[4] X. D. Hou, K. H. Leung And Xiang. Q, A generalization of an addition theorem of Kneser, Journal of Number Theory 97 (2002), 1-9. 
[5] Y. O. Hamidoune, Some additive applications of the isoperimetric approach, Ann. Inst. Fourier, 58 (2008), 2007-2036.

[6] Y. O. Hamidoune, Kneser theorem and some related questions, preprint (2010).

[7] Y. O. Hamidoune, On the connectivity of Cayley digraphs, Europ. J. Comb., 5 (1984), 309312 .

[8] Y. O. Hamidoune, On a subgroup contained in words with a bounded length, Discrete Math. 103 (1992), 171-176.

[9] X. D. Hou, On a vector space analogue of Kneser's theorem, Linear Algebra and its Applications 426 (2007) 214-227.

[10] F. Kainrath, On local half-factorial orders, in Arithmetical Properties of Commutative Rings and Monoids, Chapman \& Hall/CRC, Lect. Notes. Pure Appl. Math. 241-316 (2005).

[11] J. H. B. Kemperman, On complexes in a semigroup, Indag. Math. 18 (1956), 247-254.

[12] S. Lang, Algebra, Graduate Texts in Mathematics, Springer-Verlag New York Inc (2005).

[13] M. Madiman. A. Marcus, P. Tetali, Entropy and set cardinality inequalities for partition determined functions, with applications to sumsets, Random Structure and Algorithms, DOI: 10.1002/rsa.20385 (2011).

[14] M. B. Nathanson, Additive Number Theory: Inverse Problems and the Geometry of Sumsets, Graduate Text in Mathematics 165, Springer-Verlag New York (1996).

[15] J. E. Olson, On the sum of two sets in a group, J. Number Theory 18 (1984), 110-120.

[16] G. Petridis, New proofs of Plünnecke-type estimates for product sets in groups, preprint 2011 arXiv: 1101.3507, to appear in Combinatorica, DOI 10.1007/s00493-012-2818-5 (2013).

[17] I. Z. RuzsA, Sumsets and structure, Combinatorial Number Theory and additive group theory, Springer New York (2009).

[18] T. TAO, Non commutative sets of small doublings, preprint 2011 arXiv: 11062267 (2011).

[19] T. TAO, Sumset and inverse sumset theorems for Shannon entropy, Combinatorics Probability and Computing 19 (2010), 603-639.

[20] T. TAO, Product set estimates for non-commutative groups, Combinatorica 28 (2009), 547-594.

Laboratoire de Mathématiques et Physique Théorique (UMR CNRS 6083)

Université François-Rabelais, Tours

Fédération de Recherche Denis Poisson - CNRS

Parc de Grandmont, 37200 Tours, France.

cedric.lecouvey@lmpt.univ-tours.fr 